\title{
Technical Advances in Current PET and Hybrid Imaging Systems
}

\author{
Jae Sung Lee*
}

\author{
Departments of Nuclear Medicine, Biomedical Sciences and Brain \& Cognitive Sciences, Seoul National University, \\ Seoul, Korea
}

\begin{abstract}
The continuing efforts of investigators to achieve the best physical and clinical performances from PET (positron emission tomography) and related hybrid cameras (PET/CT and PET/MRI) have led to remarkable technical advances in hardware and software.

Time of flight (TOF) information measurement, which has been enabled thanks to the invention of fast, heavy crystals and advances in fast light detection sensors and readout electronics, is used to obtain the PET images of better quality and lesion-detection performance. Statistical reconstruction with resolution recovery and measurements of depth of interaction (DOI) are the useful software- and hardware-wise approaches for enhancing the spatial resolution of reconstructed PET images. These technical advances along with an expansive increase in the number of PET and PET/CT examinations performed are facilitating the widespread use of PET scanners with specific detector structures and arrangements for particular applications or organs. Combined multimodal imaging systems are also in the development mainstream of these PET systems.

In this paper, these important technologies (TOF measurements, statistical reconstruction with resolution recovery, DOI detectors, and application-specific PET cameras) that have made major contributions to advances in state-of-the-art clinical and preclinical PET and hybrid systems are reviewed.
\end{abstract}

Keywords: PET, time-of-flight, depth-of-interaction, resolution recovery reconstruction.

\section{INTRODUCTION}

Positron emission tomography (PET) is a major in vivo biomedical imaging modality that provides spatial information on the biochemical, functional, and molecular processes taking place in the living body. PET has unique roles during the evaluation of many diseases and is a key research tool during studies on experimental animals [1-4].

The incorporation of accurate anatomical and PET information has been made possible by combining PET with $\mathrm{X}$-ray computed tomography $(\mathrm{CT})$, and this development has made a major contribution to the expansion of PET for clinical uses (Fig. 1A) [5, 6]. In addition, the noiseless spatial information on photon attenuation provided by X-ray CT provides PET images of better image quality and quantitative accuracy and reduces PET examination times, which improves diagnostic confidence, convenience for the patient, and study throughput. Hybrid PET imaging has advanced rapidly to the integrated development of PET and magnetic resonance imaging (MRI), a major anatomical and functional imaging modality (Fig. 1B) [7-10].

The continuing efforts of investigators to achieve the best physical and clinical performances from PET cameras have led to remarkable technical advances in PET hardware and software. Thanks to the invention of fast, heavy crystals, such as, LSO and its generic versions (LYSO, LGSO, LFS,

*Address correspondence to this author at the Department of Nuclear Medicine, Seoul National University College of Medicine, 28 YungunDong, Chongno-Gu, Seoul 110-744, Korea; Tel: 822-2072-2938; Fax: 822745-2938; E-mail: jaes@snu.ac.kr and others) $[11,12]$, and advances in fast light detection sensors and readout electronics, time of flight (TOF) measurements, which used to be regarded as clinically irrelevant, have once again attracted attention for use in clinical PET cameras $[13,14]$. In addition, the hardware- and software-wise approaches (i.e., depth of interaction (DOI) measurements and resolution recovery reconstruction) used to overcome the physical limitations of PET spatial resolution and to improve the noise properties of PET images, are also being rigorously pursued and being applied to preclinical and clinical PET systems.

These technical advances along with an expansive increase in the number of PET and PET/CT examinations performed are facilitating the widespread use of PET scanners with specific detector structures and arrangements for particular applications or organs. Combined multimodal imaging systems are also in the development mainstream of these PET systems.

In this paper, I review some of the important technologies that have made major contributions to advances in state-of-the-art clinical and preclinical PET systems and PET hybrid systems. The four topics selected for review are; 1) TOF measurements, 2) statistical reconstruction with resolution recovery, 3) DOI detectors, and 4) applicationspecific PET cameras.

\section{TIME-OF-FLIGHT PET/CT AND PET/MRI}

\section{Principles of TOF PET}

TOF measurements represent one of the most important technical advances in the clinical PET/CT instrumentation field [13]. The two $511 \mathrm{keV}$ gamma rays emitted when a 


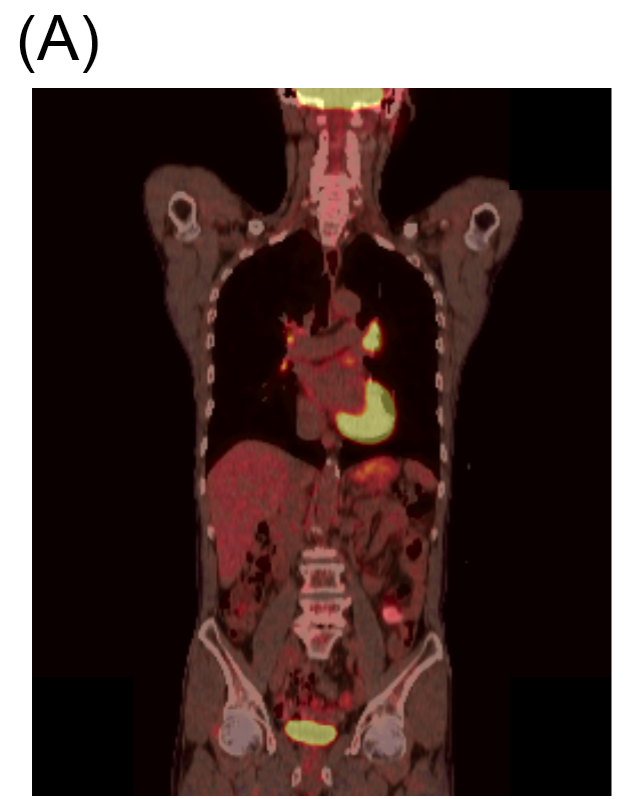

(B)

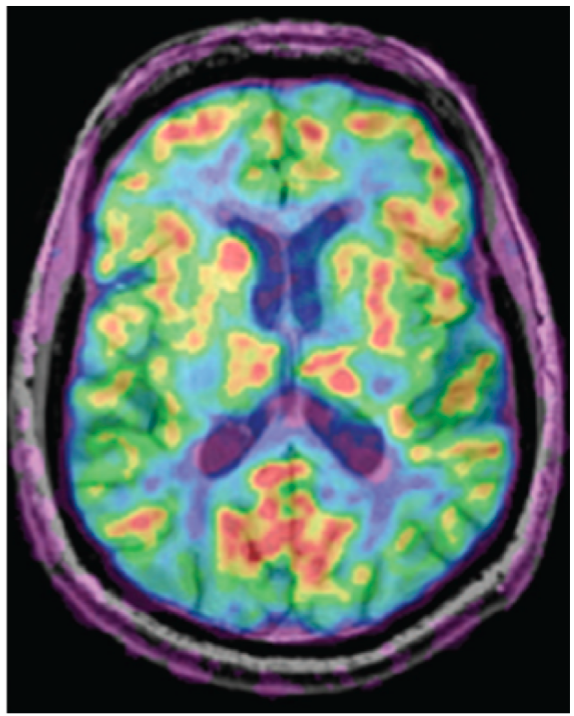

Fig. (1). Hybrid PET images. (A) PET/CT. (B) PET/MRI (Reprinted from [7]).

positron and electron mutually annihilate each other arrive at PET detectors at different times if they have not been generated at the exact center of two detectors. Furthermore, if we could measure differences between arrival times precisely, we could pinpoint annihilation positions. The annihilation positions of gamma ray pairs (distances from the geometric center to annihilation positions) can be determined using $d=c\left(t_{1}-t_{2}\right) / 2\left(c\right.$ : speed of light $=3 \times 10^{8} \mathrm{~m} / \mathrm{sec}, t_{1}-t_{2}$ : difference between the arrival times of gamma rays) (Fig. 2A). Accordingly, given ideal conditions, we do not need to use any complex image reconstruction algorithm based on inverse problem solving techniques to obtain tomographic PET images.

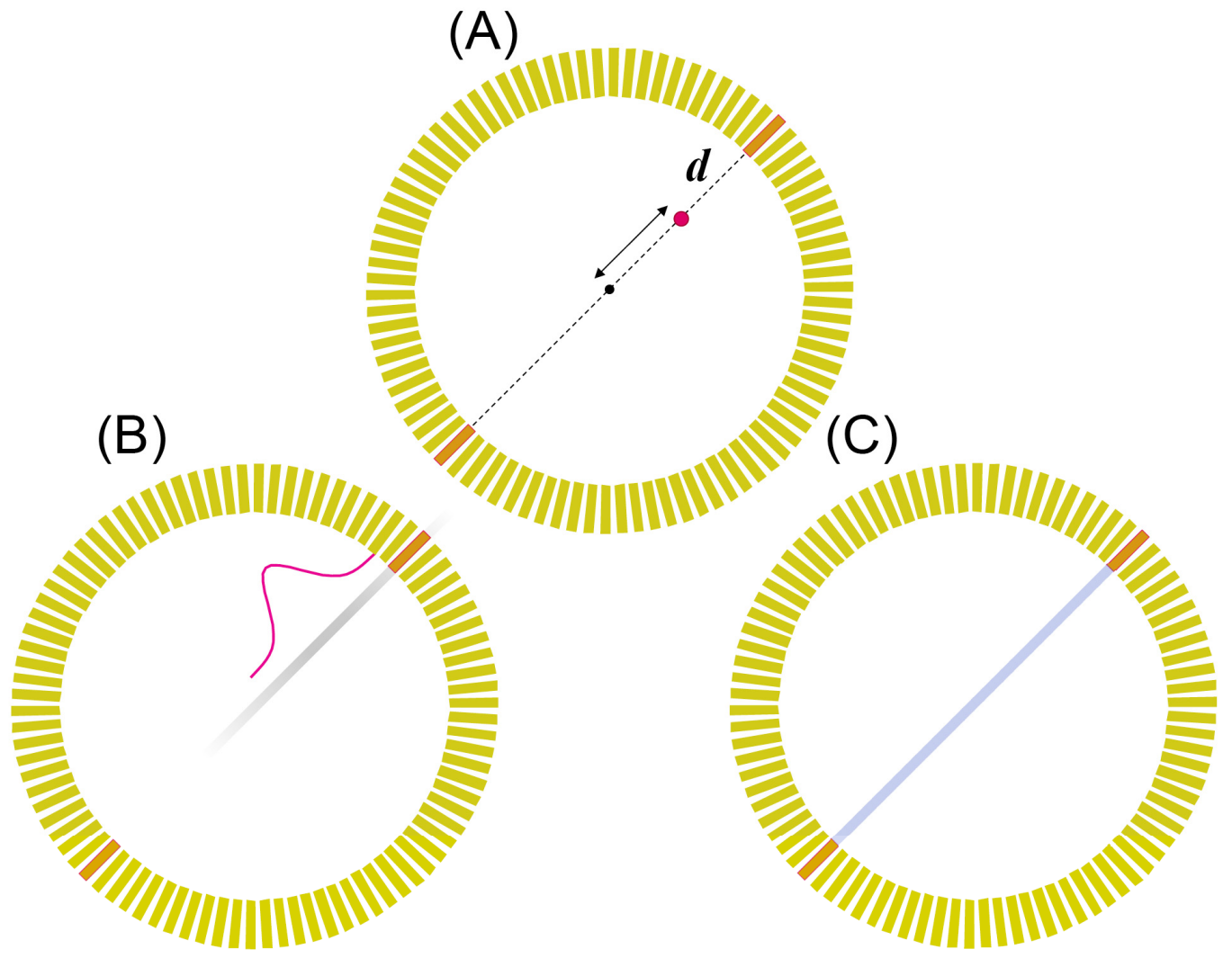

Fig. (2). Uncertainty of annihilation position in PET (A) with accurate time of flight (TOF) information. (B) With uncertain TOF information. (C) Without TOF information. 

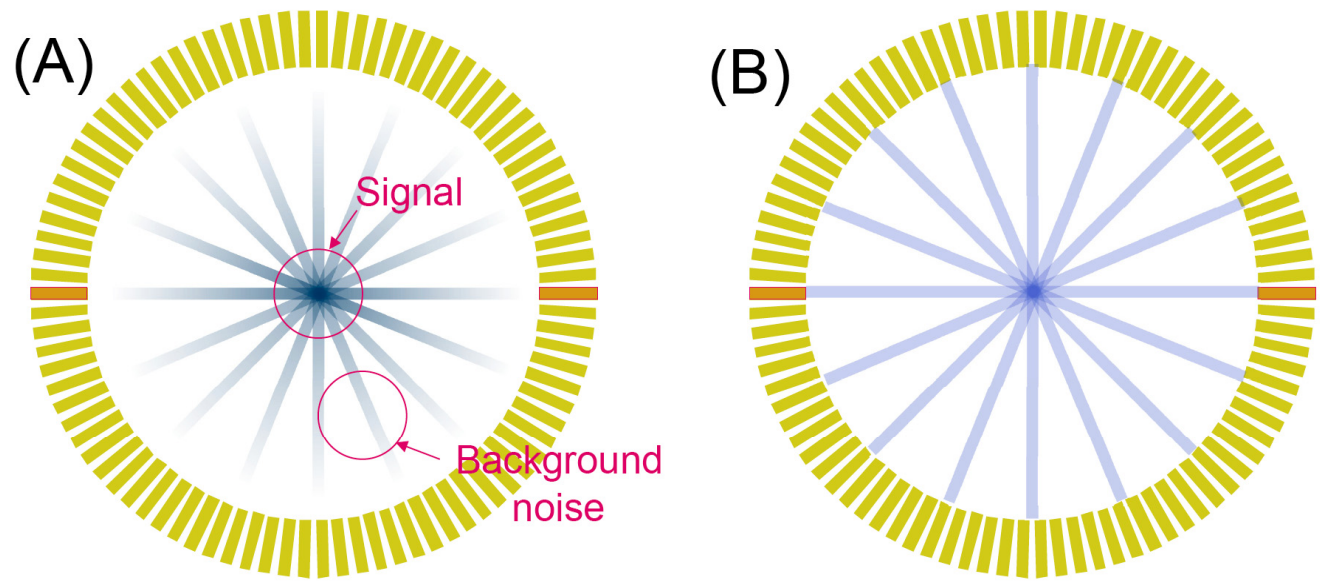

Fig. (3). Background noise propagation in (A) TOF reconstruction and (B) conventional non-TOF reconstruction.

However, the timing resolutions of currently available clinical PET detectors based on cerium-doped lutetium silicates (i.e., LSO and LYSO) are of the order of 600 picoseconds (FWHM) [14], which equates to a positional uncertainty of $\sim 9 \mathrm{~cm}$, which is not low enough to provide clinically relevant PET images based on positional information alone (Fig. 2B).

However, TOF information can be used to reduce background noise in back-projection based reconstruction images. This is because the back-projected events can be confined within areas around estimated annihilation positions, as shown in Fig. (3A), which can then be compared with back-projection without TOF information, as shown in Fig. (3B).

\section{Benefits of TOF PET}

Improvements in signal to noise ratio (SNR) achieved by incorporating TOF information (amount of variance reduction) are described by:

$$
f=\frac{2 D}{c \cdot \Delta t}
$$

where $D$ is the size (diameter) of the emission source and $\Delta t$ is the coincidence timing resolution of the TOF PET system (i.e., 3.9 for $\mathrm{D}=35 \mathrm{~cm}$ and $\Delta t=600 \mathrm{ps}$ ) [15].

Thanks to the SNR enhancements during TOF PET reconstruction, we can obtain PET images of better quality and lesion-detection performance, or reduce either scan times or the radiopharmaceutical doses administered, as illustrated in Fig. (4) $[16,17]$. This would be especially beneficial in large patients, because SNR improvements are proportional to object size $(D)$, as shown by the above equation. In oncologic FDG PET studies of heavy patients (weight $\geq 115 \mathrm{~kg}$, BMI $\geq 38$ ), images reconstructed using TOF showed better structural details and depicted lesions more clearly with higher uptake than images reconstructed without TOF (Fig. 5) [16]. More benefits, in terms of contrast-to-noise ratio (CNR), can be obtained in central and low- or medium-count regions than in eccentric and high-



Fig. (4). Benefit of TOF PET in the image quality and scan time assessed with 35 -cm-diamter phantom with 2 cold and 4 hot spheres (Reprinted from [16]). 


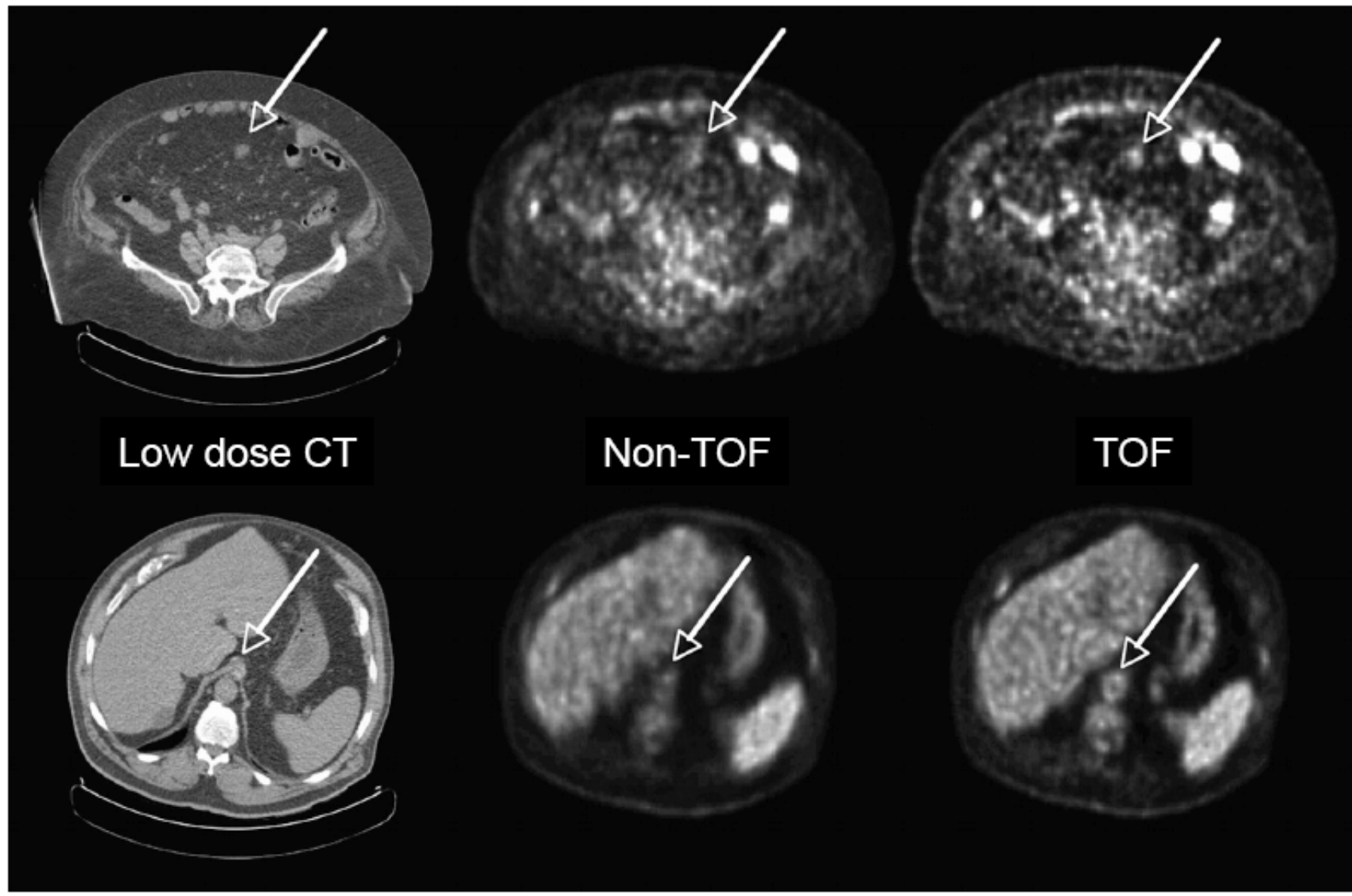

Fig. (5). Benefit of TOF in FDG PET studies of heavy patients. Better lesion detection performance can be obtained in TOF PET relatively to non-TOF PET (Reprinted from [16]).

count regions [18]. In addition, increased patient throughput due to reduced scan times, and reduced radiation exposure achieved by reducing radiopharmaceutical doses are important benefits of TOF measurements from the practical and ethical viewpoints. The potential usefulness of TOF measurement in I-124 PET, which has attracted much interest for dose monitoring in radio-immunotherapy because of its long half-life (4.2 days) [19], is also evident, because the improved SNR in TOF PET can compensate for reduced I-124 PET sensitivity caused by its low branching ratio for positron emission (0.23) [20].

\section{TOF and Limited Angle PET}

TOF information is also useful for reducing reconstruction artifacts in limited angle PET cameras. Although a solid ring with full angular coverage is the most common PET geometry, partial ring configurations are also used when the open PET geometry is preferable, because it allows easier patient access. Partial and split ring configurations for breast dedicated PET enable PET-guided biopsies to be performed on suspicious breast tissues [21]. Such open PET geometries are also attractive for those developing in-beam PET scanners to monitor $\beta^{+}$activity distributions generated by charged-hadron irradiation for radiation therapy [22]. In this specific application, closedring PET detectors pose spatial challenges if the horizontal and rotating radiotherapeutic beam lines are utilized. Furthermore, partial ring systems with longer axial rings are more beneficial than full ring systems with respect to maximizing geometric detection sensitivity for a given number of detectors [23].

However, despite these advantages of partial ring PET geometry, its use has been limited because it is prone to serious reconstruction artifacts due to limited angular sampling. Although detectors can be rotated to obtain complete data and prevent these artifacts, the implementation of rotating detectors is technically challenging, and undermines the merits of the open PET geometry. On the other hand, TOF information allows limited-angle artifacts to be reduced without incorporating detector rotation, because the back-projection of data is restricted in a narrow region, for example, the elongation of image intensity in a direction perpendicular to the detectors in non-TOF PET images can be reduced using TOF information (Fig. 6) [21-23]. Coincidence timing resolution is an important determinator of the degree of artifact reduction, and individual partial ring configurations have different required timing resolution levels.

\section{Potential of TOF PET/MRI}

PET/MRI scanners with TOF measurement capability will have the same advantages as TOF PET/CT. However, the implementation of MR-compatible PET systems with sufficient timing resolution for TOF measurements is technically challenging because the photomultiplier tube (PMT) that is conventionally used as a photo-sensor for PET scintillation detectors does not operate properly in the presence of a magnetic field. In spite of this incompatibility 


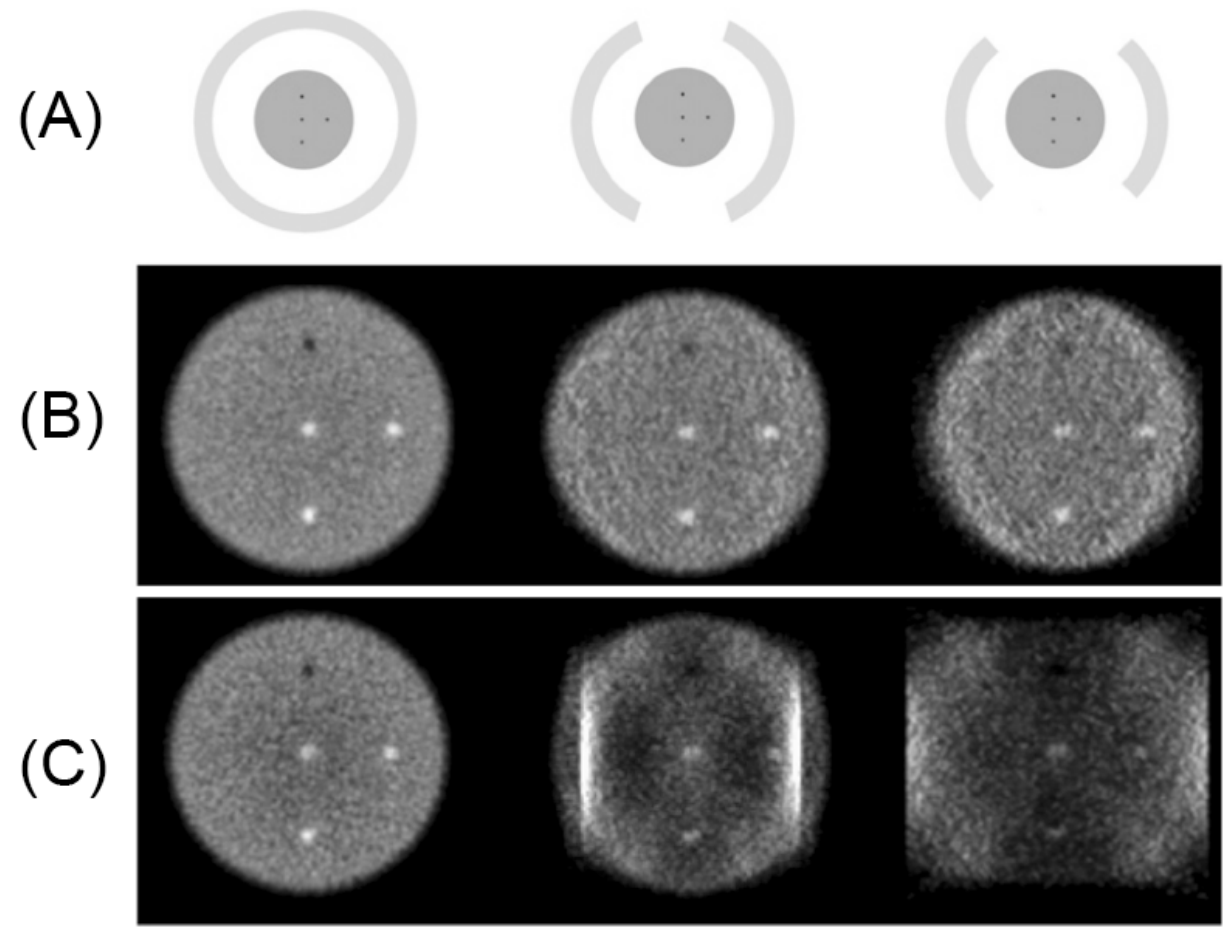

Fig. (6). TOF measurement in limited-angle PET. (A) Detector arrangements for complete (left) and limited-angle partial ring (middle and right) ring PET cameras. (B) Reconstructed image with TOF information (timing resolution $=200 \mathrm{ps}$ ). (C) non-TOF reconstruction. (Reprinted from [21]).

between PMT and magnetic fields, PMTs have several advantages over other photo-sensors, e.g., relatively high gain, fast response, and low cost. In addition, PMTs better tolerate changes in operating conditions. Based on the proven merits of PMTs, Philips (the company that introduced the first commercial TOF PET/CT scanner) is developing a PET/MRI system in which the PMT-based TOF PET scanner is combined with a 3.0T MRI machine (Fig. 7). The TOF PET and 3.0T MRI scanners are operated in the same scanning room, in which they are separated by a face-to-face distance of $\sim 2.5 \mathrm{~m}$ and a rotating bed placed between the scanners is used to present patients to the scanners. This approach is made possible because a selfshielded MRI machine is used, which reduces the magnetic field to less than 50 Gauss at the PET gantry. In addition, shielding materials are used to protect individual PMTs and the PET gantry.

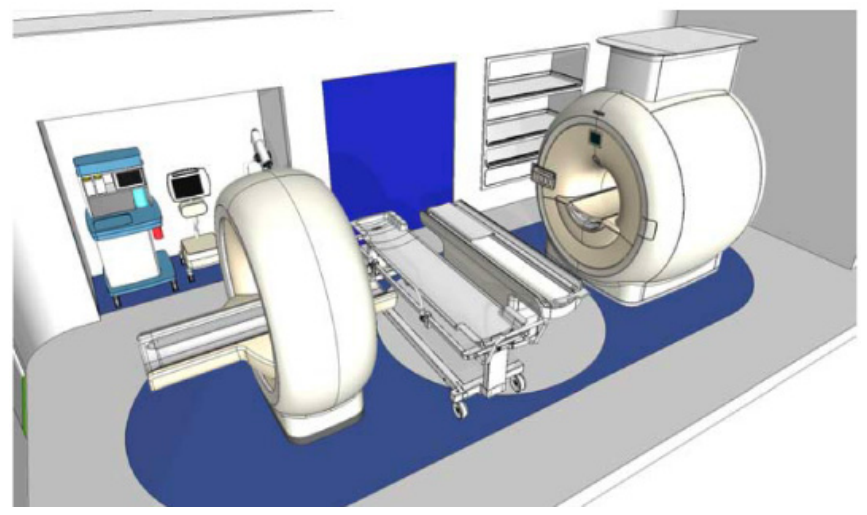

Fig. (7). Design concept of TOF PET/MRI with separate scanners suggested by Philips. PMT-based TOF PET scanner is combined with state-of-art 3.0T MRI device (Courtesy of Philips Medical).
PMT-based PET detectors have limited performance for the fully integrated PET/MRI scanners designed for simultaneous $\mathrm{PET} / \mathrm{MR}$ imaging that does not require the patient transfer between the scanners. For this approach, PMTs are coupled to optical fibers that transfer visible light photons generated by scintillation crystals located inside the MRI magnet, and thus, can be operated in environments isolated from strong magnetic fields [24-27]. However, the significant light loss that occurs during optical transfer leads to a degradation of timing resolution, and thus, it is suspected that PMT/optical fiber approaches are likely to be unsuitable for TOF PET/MRI scanners [28].

Although simultaneous PET/MRI scanners utilizing avalanche photodiodes (APDs) have been successfully applied during the development of preclinical and clinical MR-compatible PET detectors [7, 29, 30], the use of APDs is not likely to provide TOF capability. Although APDs have several advantages, such as, a compact size and insensitivity to magnetic fields, the major concern regarding the use of APDs for TOF measurement is that the timing resolutions of the array and position-sensitive types are limited [31].

On the other hand, Geiger-mode APDs (G-APDs), a relatively new type of semiconductor photo-sensor, are more promising than conventional APDs for MR-compatible TOF PET detectors $[10,31]$. Although they are referred to using different names by different manufacturers, such as, SiPMs, SSPMs, and MPPCs, G-APDs have a common structure of parallel inter-connecting micro APD cells operated in Geiger-mode (Fig. 8). The amplitudes of output signal from G-APDs are proportional to the number of micro cells activated by the individual incident photons, which enables spectroscopy to be used to measure the energies of detected gamma rays (Fig. 9) [32, 33]. In addition to the exceptional 

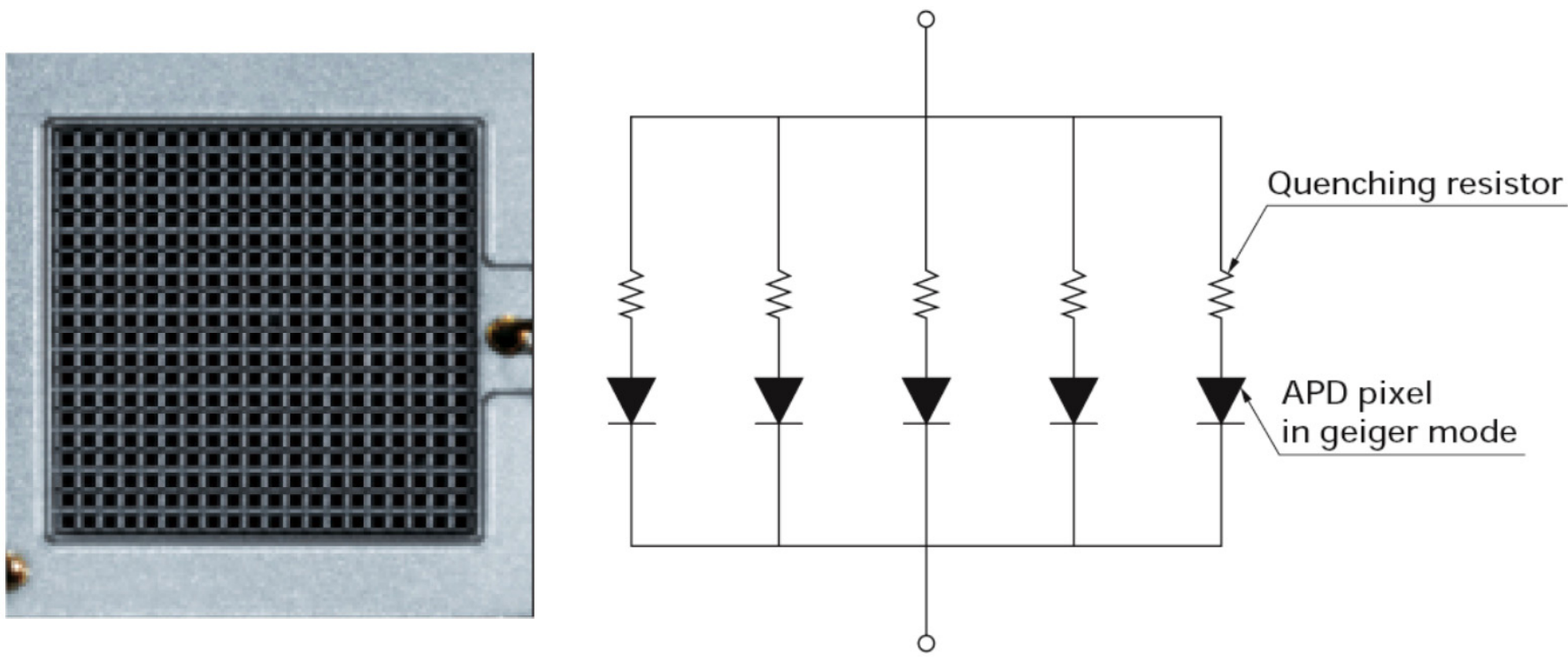

Fig. (8). Structure of Geiger-mode APD (G-APD): micro APD cells operated in the Geiger-mode are inter-connected in parallel way (Courtesy of Hamamatsu Photonics).

high signal amplification $\left(10^{5} \sim 10^{6}\right)$ achieved, G-APDs have good timing resolutions, which positions them well for use as photo-sensors in TOF-capable, fully integrated PET/MRI scanners. For example, two LYSO/MPPC detectors yielded a coincidence timing resolution (at FWHM) of $240 \mathrm{ps,} \mathrm{which}$ compared well with that of a fast PMT (Fig. 10) [34].

\section{RESOLUTION RECOVERY RECONSTRUCTION}

\section{Resolution Degrading Factors in PET}

Several factors determine the spatial resolutions of PET scanners. High energy $511 \mathrm{keV}$ gamma rays require a relatively large detector element as compared with X-ray CT, which detects relatively low energy photons. Positron range (the distance traveled by a positron before it undergoes annihilation) and displacement errors, which are caused by the non-collinear annihilation of gamma ray photons, also degrade spatial resolution (Fig. 11). Furthermore, spatial resolutions are further reduced when light signals from scintillation crystal arrays are not read individually, but rather multiplexed using the light sharing or resistive charge division methods to reduce the number of readout channels.

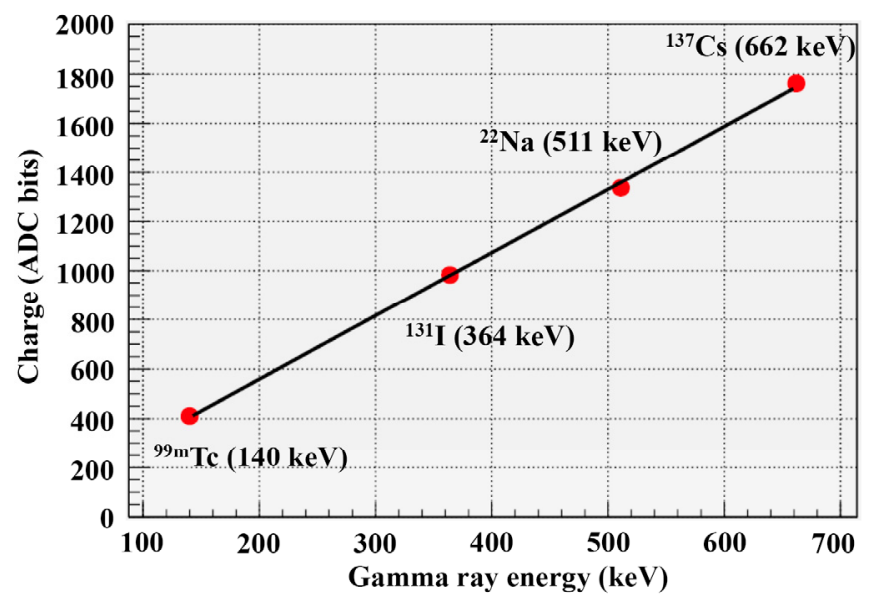

Fig. (9). Response of G-APD that is proportional to the gamma-ray (Reprinted from [33]).
At the peripheral region of the FOV in ring type PET scanners, parallax error makes the spatial resolutions worse and non-uniform (Fig. 12). In ring type PET cameras, gamma ray pairs emitted from a position far from the center of ring (at the edge of the FOV) enter scintillation crystals at significant angles of incidence. Because the front surface areas of crystals in PET cameras are small $\left(1 \times 1 \mathrm{~mm}^{2}\right.$ for small animal $\sim 4 \times 4 \mathrm{~mm}^{2}$ for human), to obtain sufficient spatial resolution, the path length of gamma rays in individual crystals is relatively short as compared with the attenuation length of $511 \mathrm{keV}$ gamma rays (i.e. $11.4 \mathrm{~mm}$ in LSO), and as a result, significant numbers of photons cannot be captured by the first-met crystal, and incorrect positioning

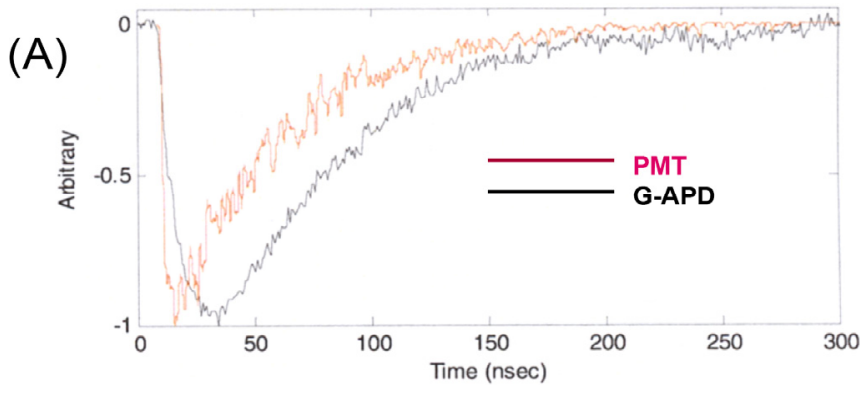

(B)

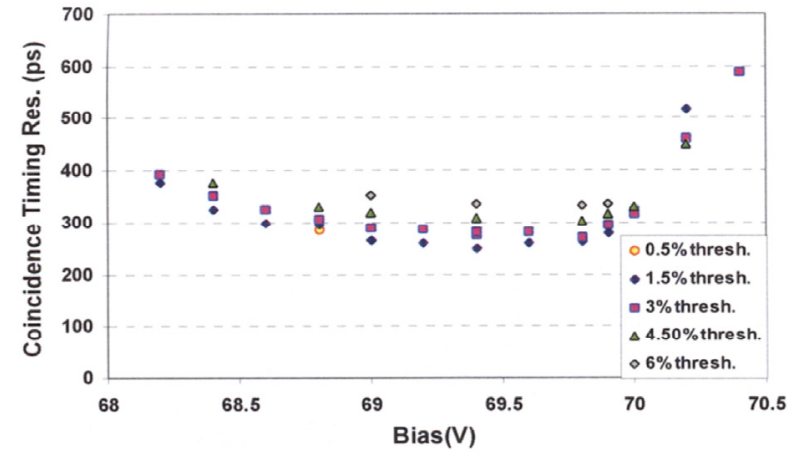

Fig. (10). Timing properties of G-APD. (A) Output pulses from PMT and G-APD. (B) Coincidence timing resolutions measured with LYSO/G-APD pairs. (Reprinted from [34]). 


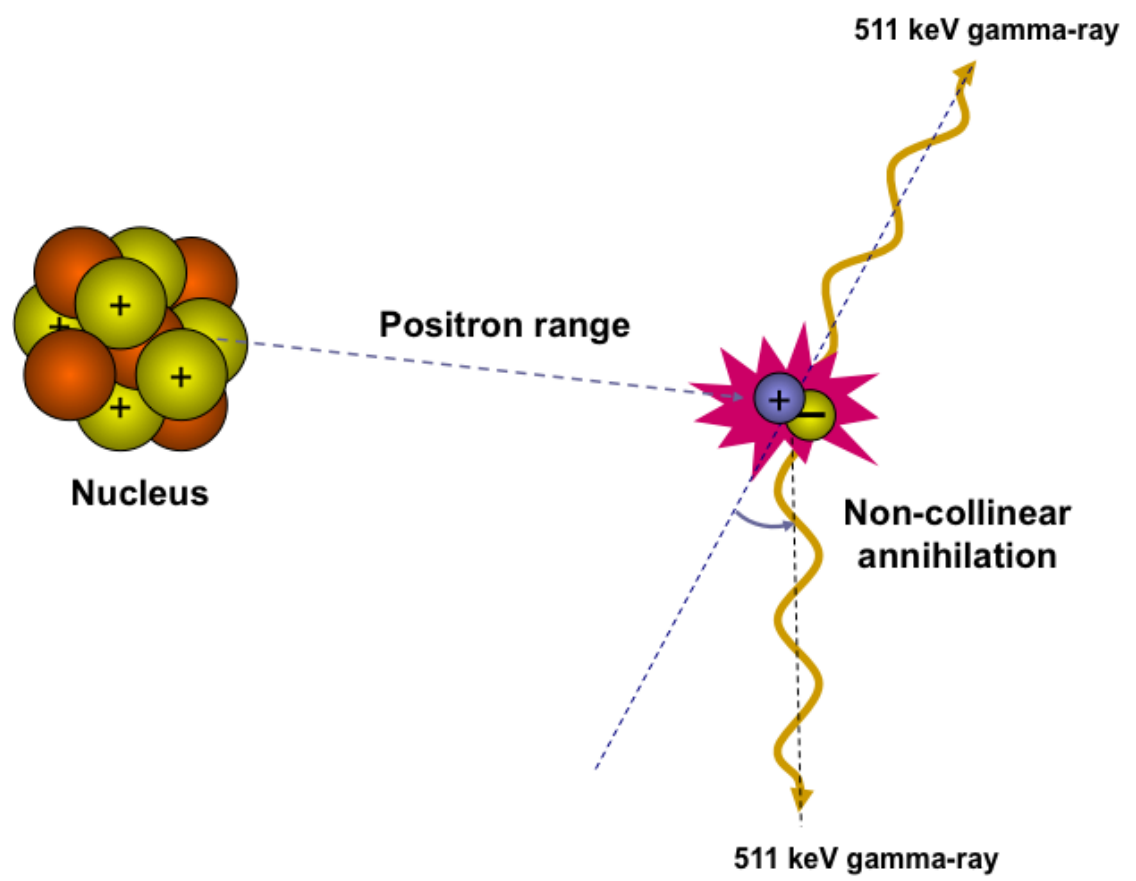

Fig. (11). Positron range and non-collinear annihilation: degradation factors for PET spatial resolution (Reprinted from [101]).

of the interaction point as a consequence of crystal penetration (parallax error) is possible (as illustrated in Fig. 12). Furthermore, the use of longer crystals to enhance sensitivity increases parallax errors, which affect a large proportion of FOVs in small ring PET cameras, such as, small animal dedicated scanners. Therefore, the spatial resolution of PET is limited and not uniform across the FOV. Although some optimal filters, such as, the Metz and Wiener filters are used for resolution recovery in gamma camera or SPECT images, they are rarely employed during modern PET data processing.

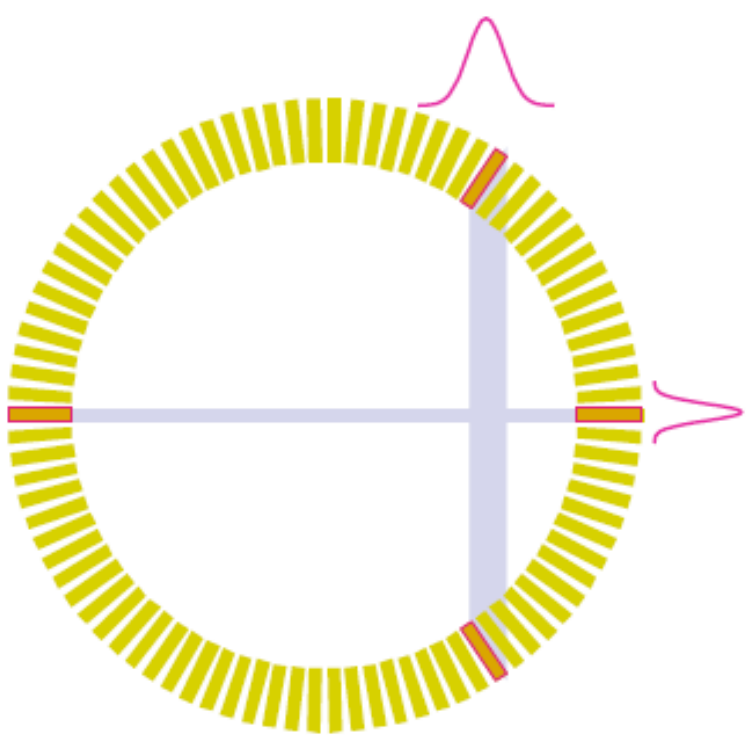

Fig. (12). Parallax error at the peripheral region of the FOV in ring type PET camera (Reprinted from [101]).

\section{Resolution Recovery Modeling}

Iterative statistical reconstruction is now a standard PET reconstruction method for clinical PET data, and spatial resolution can be enhanced by incorporating a resolution degradation model in the iterative reconstruction procedure (Fig. 13). The accurate description of the relationship between the image and projection spaces by a system matrix (each element of the matrix represents the degree of association between each image voxel and each projection element) is an important factor that determines the image quality achieved by statistical reconstruction [35]. In conventional method, the system matrix is determined using the intersection lengths or areas between lines of response (LOR) and voxels [36-38]. However, these methods are not accurate enough to describe this relationship, because resolution degradation due to the above-mentioned physical factors is not considered.

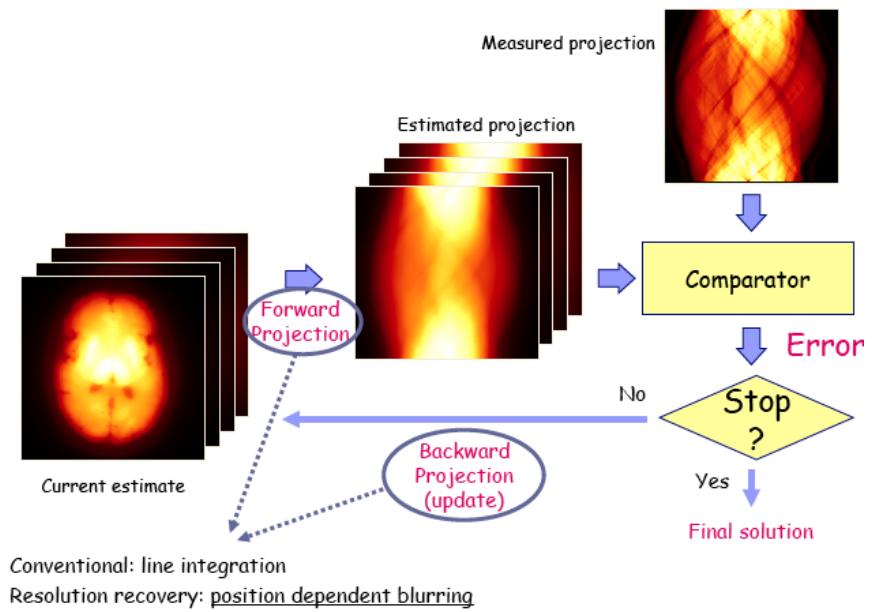

Fig. (13). Iterative statistical reconstruction incorporating a resolution degradation model (Modified from [101]).

To derive a more realistic system matrix that incorporates degradation factors, several methods have been suggested and used, such as, analytical calculations, Monte Carlo simulation, and point source measurements [35, 39-41]. 
Although this resolution modeling is usually performed in projection space, modeling in image space is possible and can be implemented fairly easily [42].

These improved system modeling approaches both enhance spatial resolution and reduce statistical noise (at the expense of computation time) (Fig. 14). Accordingly, the improved lesion to background contrast and lesion detection performance can be achieved (Figs. 15, 16) [42, 43]. In addition, the improved spatial resolution using these techniques contributes to the enhancement of quantitative accuracy particularly in small lesions. Although the improved spatial resolution and image quality obtained using this approach increase computation time due to the complexity of the system matrix, fast reconstruction techniques based on advanced modern computer technology using CPU clusters and/or graphic processing units (GPU) allows clinically relevant reconstruction times [44-49].

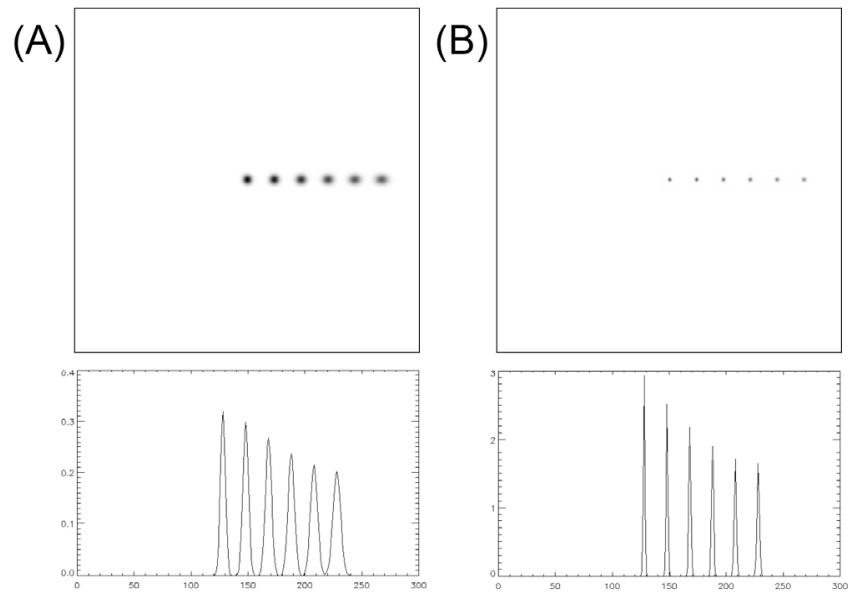

Fig. (14). Effects of resolution recovery reconstruction: reconstructed images of the point sources (A) without and (B) with resolution recovery modeling.

\section{Hybrid Approaches}

Further improvement in the benefits of resolution recovery reconstruction can also be achieved by combining this method with other hardware-driven advanced technologies, such as, those that provide DOI position measurements and TOF information [13, 50]. In a recent study, Kadrmas et al. [13] showed that when TOF information is utilized in conjunction with resolution recovery modeling, lesion detection performances are improved both in numerical and human observer tests as compared with either technique resolution recovery or TOF alone (Fig. 17).

\section{DEPTH-OF-INTERACTION MEASUREMENTS}

DOI measurements provide another useful means of improving detection sensitivity and spatial resolution, especially those of small bore ring-type PET cameras (e.g., brain, breast, and animal PET) because the parallax error mentioned above can be corrected using DOI information (Fig. 18). Many creative suggestions have been regarding the measurement of DOI in scintillation detectors. These methods can be divided into two approaches.

\section{Discrete DOI Encoding}

Discrete DOI encoding involves the recoding of DOI data using multiple discrete layers of crystal blocks. These approaches can be further categorized based on the method used to identify the crystal layer containing individual gamma ray interactions.

The most direct method involves reading the light output from each crystal block using thin semiconductor photosensors (e.g., avalanche photodiodes (APDs)) (Fig. 19A) [51]. Although this method provides DOI information intrinsically, the large numbers of photo-sensors required increase development and manufacturing costs.

DOI information can be also obtained using light emitted from the rear surfaces of stacked, optically coupled, multiple crystal blocks [52-57]. This method offers cost advantages over the above-mentioned direct readout from crystal layers method, because no additional photo-sensors are required. According to this approach, two or more layers of pixelated crystals with different scintillation decay times are stacked and the shapes of output pulses obtained from photo-sensors

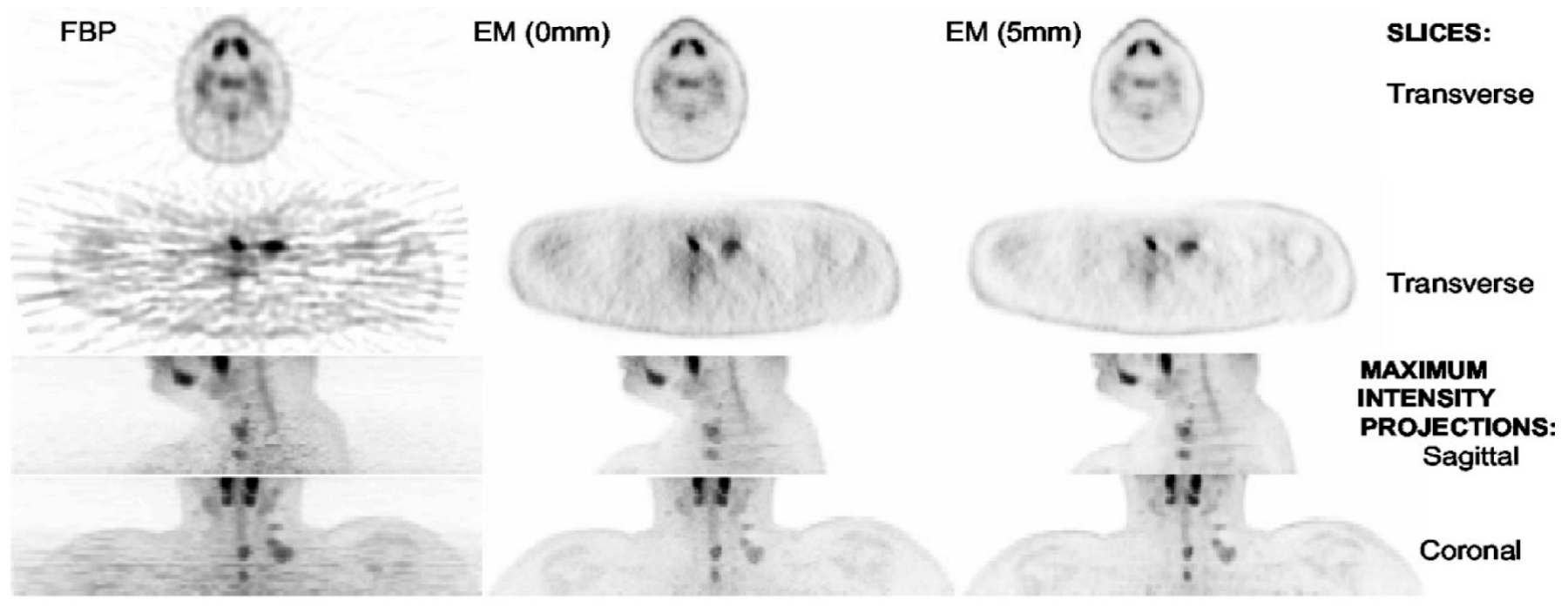

Fig. (15). Resolution recovery reconstruction in a clinical case (head and neck cancer patient). Images in the right column were reconstructed using resolution recovery modeling and compared with the conventional methods (left and middle). (Reprinted from [42]). 
coupled to these crystals are analyzed to determine the interaction position (so-called pulse shape analysis or pulse shape discrimination) (Fig. 19B). This DOI encoding method has been applied to several commercialized DOI PET scanners. In the latest commercial version of the braindedicated HRRT scanner, 10-mm long LSO and LYSO crystals are employed with decay times of 40 and $53 \mathrm{~ns}$, respectively.
(A)

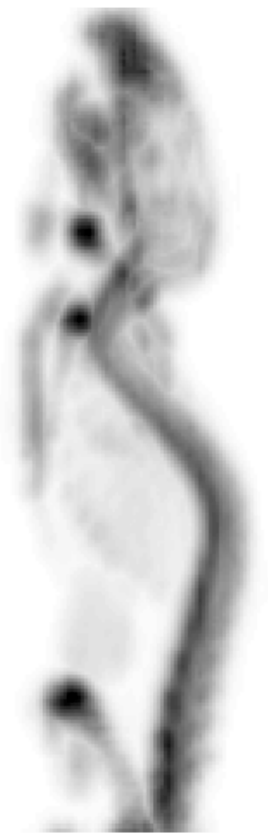

(B)

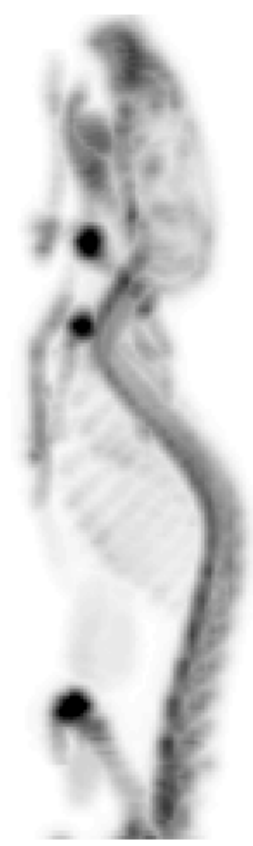

Fig. (16). Projection images of mouse skeletal F-18 PET reconstructed (A) without and (B) with resolution recovery modeling. (Reprinted from [43]).
(A)

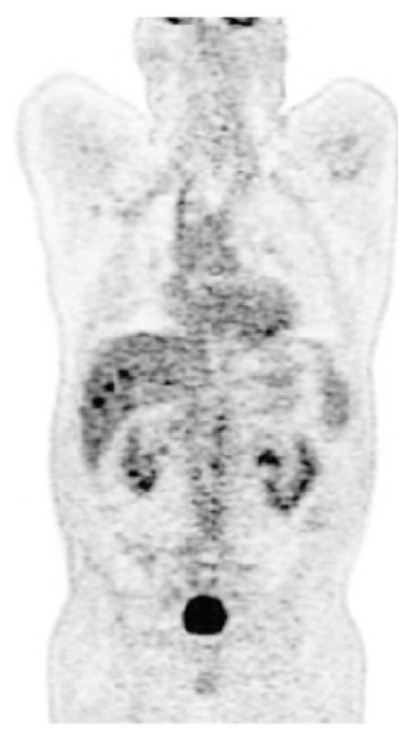

(B)



Fig. (17). Conjunction of TOF information with resolution recovery (RR) modeling. (A) RR only. (B) RR+TOF. (Reprinted from [13]).

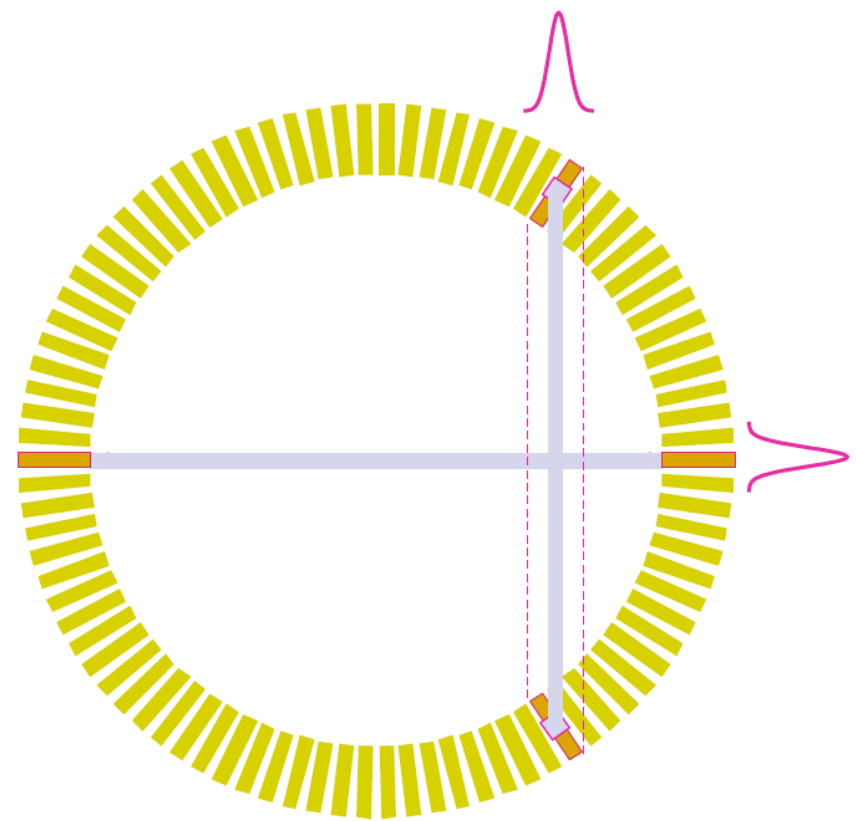

Fig. (18). Enhancement of spatial resolution using DOI information. (Reprinted from [101]).
(A)

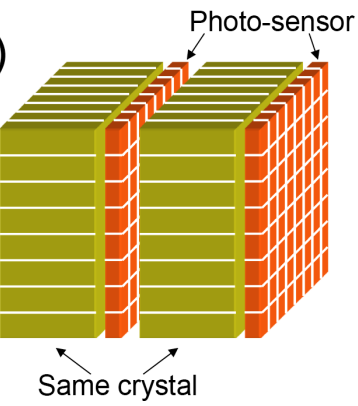

(C)

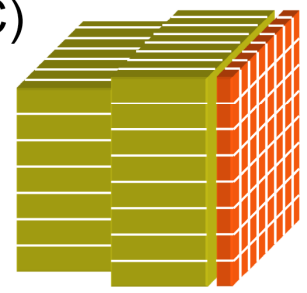

(B)

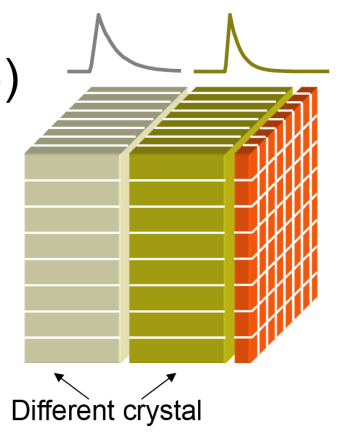

(D)

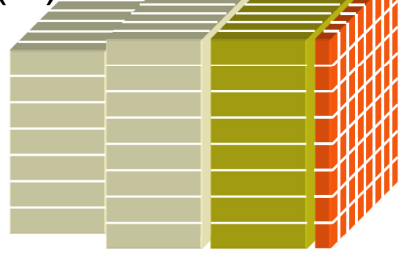

Fig. (19). Discrete DOI encoding methods. (A) Light readout from each individual crystal layer. (B) Pulse shape discrimination. (C) Relative offset method. (D) Combination of (B) and (C).

In the GE eXplore VISTA, a commercial version of the ATLAS animal PET scanner developed by the NIH group, LYSO (40 ns decay time) and GSO (60 ns) crystals of total length $15 \mathrm{~mm}(7+8)$ are used [54]. For pulse shape discrimination, the so-called delayed charge integration method is used, whereby the last dynode signal of the photomultiplier tube is split into two parts. One of these parts is fully integrated from the start for an integration period (full), whereas the other is integrated after a delay for equal integration period (delayed) [53]. Crystals with different decay times have different delayed/full ratios, which enables DOI discrimination. In the LabPET scanner, 
the PET component of the Triumph pre-clinical SPECT/PET/CT system produced by GE, LYSO and LGSO crystals coupled to APD detectors are employed, and digital signals are sampled using fast free-running ADC and analyzed using an adaptive filtering scheme to discriminate the interaction position [55]. In addition, to the abovementioned commercialized scanners, the feasibility of the pulse shape discrimination method has been confirmed by several other research groups [56, 57].

The relative offset method provides another means of obtaining DOI information using stacked crystal layers and a single-end readout. According to this approach, one of two crystal layers is shifted by half a crystal pitch in both the horizontal and vertical directions (Fig. 19C) [58, 59]. Visible light photons emitted from the crystals are read by positionsensitive PMT or by multi-channel PMT, which provide the centroid of the light distribution, which is also shifted between the crystal layers. Accordingly, 3D positional information of a gamma ray interaction can be obtained from the 2D flood map of the PMT output (spatial distribution of the centroid of each event). The Shimazu Clairvivo small animal PET employs this method to improve the sampling rate in the axial direction [60]. A four layer configuration of this approach is also possible, and is achieved by shifting the second and third layers only in the horizontal and vertical directions, respectively, and the forth layer in both directions relative to the first layer [61]. A similar light spreading method, in which the centroid of light spreading is shifted in the same way without offsetting a crystal layer has also been applied to the development of a brain-dedicated PET (jPETD4 of the NIRS, Japan) [41, 62].

By combing the above-mentioned approaches, crystal layer numbers can be further increased (Fig. 19D). Hong et al. demonstrated the feasibility of combining the relative offset method and the pulse shape discrimination method for 3 or 4-layer DOI detectors [57]. Eight-layer DOI detectors could be also devised by combining the 4 layer encoding method, the relative offset or jPET method, and the pulse shape discrimination method [63].

\section{Continuous DOI Encoding}

Continuous DOI encoding within scintillation crystals offers another means of DOI encoding. This method does not require multiple layers of crystals, and thus, is cheaper in terms of crystal costs and crystal block assembly. Several different continuous DOI encoding schemes have been proposed.

One of the most popular methods involves the signal readout of scintillation light from both ends of a pixelated or discrete crystal (a narrow, long crystal element of a crystal block) (Fig. 20A) [64-66]. The ratio of the signals obtained from both ends is compared to determine the interaction position within the crystal. Because the closer detector allows higher geometric efficiency and the light is partly absorbed by the scintillation crystal itself, higher signal amplitudes are obtained at ends closer to gamma ray interaction positions. Solid-state photo-sensors are commonly used for light readout from the front surfaces of crystals directed toward the object. In a recent study, a DOI resolution of $\sim 2 \mathrm{~mm}$ was obtained using this approach and a
2 position-sensitive APD and LSO crystal block with $\sim 1 \mathrm{x} 1$ x $20 \mathrm{~mm}^{3}$ crystals [66].

DOI estimations from arrays of discrete crystals using the single-end readout scheme are challenging, but offer an attractive approach, because the same numbers of crystals and photo-sensors are required as in conventional non-DOI PET detectors. The Washington University group proposed a single-end readout method in which they estimated DOI information based on light sharing along the long length of a pair of crystals $[67,68]$. Using this approach, all non-shared long surfaces are covered by reflective material and light sharing between these long surfaces is tailored so that less light sharing occurs when the interaction position of a gamma ray in a crystal is closer to the surface of the photosensor. Therefore, the ratio of light output from photosensors attached to only one end of crystals provides DOI information. Recently, the Seoul National University group devised a 2-directional (2D) light spreading method using a multi-channel PMT to measure light spreading in discrete crystal blocks. Accordingly, to this method, both the extent and direction of the 2D light dispersion is shaped using a reflector grid structure with triangular teeth, which enables DOI measurements at resolutions of better than $2 \mathrm{~mm}$ for a crystal block with $1.5 \times 1.5 \times 20 \mathrm{~mm}^{3}$ crystals [69].

The extent of light dispersion in a monolithic crystal (crystal slab) also provides DOI information in a continuous manner (Fig. 20B). Because only the single crystal slab is used, manufacturing costs are lower than approaches that use discrete crystals [70, 71]. Although simple centroid positioning algorithms (e.g., Anger logic) have limited performances in terms of intrinsic spatial resolution, positional linearity, and effective FOV, they could be improved by using statistical estimation algorithms (e.g., the maximum likelihood estimation) [72].

Quasi-monolithic crystal arrays use a combination of monolithic and discrete crystals [73]. Accordingly, to this method, crystal elements are separated from each other in the axial direction but are monolithic in the trans-axial direction (Fig. 20C). Because only trans-axial and DOI positions should be estimated from monolithic crystals, positional accuracy and DOI estimations may be better than those determined using the pure monolithic crystal approach. The same group recently proposed cross-stacking two layers of quasi-monolithic crystal arrays to extend the total crystal thickness to $20 \mathrm{~mm}$, while maintaining the advantages of a quasi-monolithic detector, and achieved a spatial resolution of 2-mm and 2-bit DOI encoding [74].

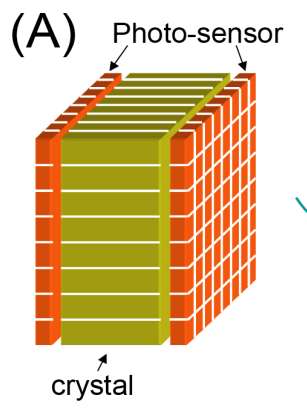

(B)

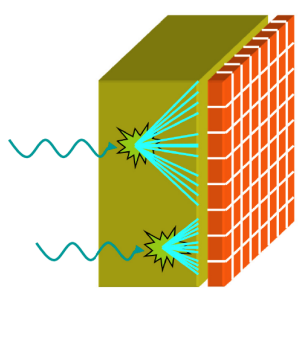

(C)

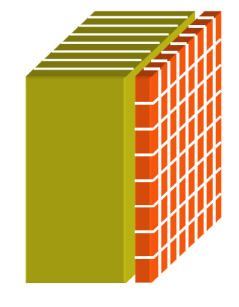

Fig. (20). Continuous DOI encoding methods. (A) Dual-end light readout. (B) Monolithic crystal slab. (C) Quasi-monolithic crystal array. 


\section{APPLICATION-SPECIFIC SYSTEMS}

It is likely that application-specific PET scanners, which are designed for specific applications or organs to maximize PET performance, will be increasingly used to assess patients. Before PET was generally available for clinical use, few PET or PET/CT scanners were available in nuclear medicine departments or PET centers even at university hospitals, and these were used for various clinical purposes and for research studies. Whole-body PET scans for cancer detection are the most frequently performed studies. Thus, the bore sizes of conventional (whole body) PET/CT scanners are sufficiently large to scan any part of the body and can be optimized with respect to torso size. However, as the numbers of PET studies conducted are increasing due to the wide recognition of the usefulness of PET for clinical work-up, smaller and larger PET scanners than the conventional one are beginning to catch on as new diagnostic tools.

\section{Brain-Dedicated PET}

Brain-dedicated PET scanners are design to provide detailed anatomical information and improved quantitative accuracy. They have smaller ring diameters $(40 \sim 50 \mathrm{~cm})$ and crystal sizes $(2 \sim 4 \mathrm{~mm})$ than whole-body PET/CT scanners to increase spatial resolution, sensitivity, and image quality (Fig. 21) [41, 52, 75-77]. For example, the Siemens HRRT PET scanner has a ring diameter of $46.9 \mathrm{~cm}$ and an axial field-of-view (FOV) of $25.2 \mathrm{~cm}$, a transaxial spatial resolution of below $2.5 \mathrm{~mm}$ (when images were reconstructed using the OSEM algorithm), and an absolute line sensitivity of above $2.5 \%$ (when the energy window is set at $400 \sim 650 \mathrm{keV}$ ) at the center of a FOV $[78,79]$. In addition, its resolution uniformity at the periphery of its FOV is much better than those of whole-body PET scanners because dual layer scintillation crystals with different scintillation decay times (LSO and LYSO in the latest HRRT version) provide information on the DOI of each incident gamma ray in the crystal block.

The improved ability of brain-dedicated PET scanners to identify small brain structures and to assess regional brain activity reliably has been demonstrated (Fig. 22) [80, 81]. However, despite these advantages, the clinical uses of brain-dedicated PET or PET/CT scanners have been limited primarily due to their marginal cost-effectiveness in the hospital environment. However, it should be noted that the potential for the clinical use of brain-dedicated PET or
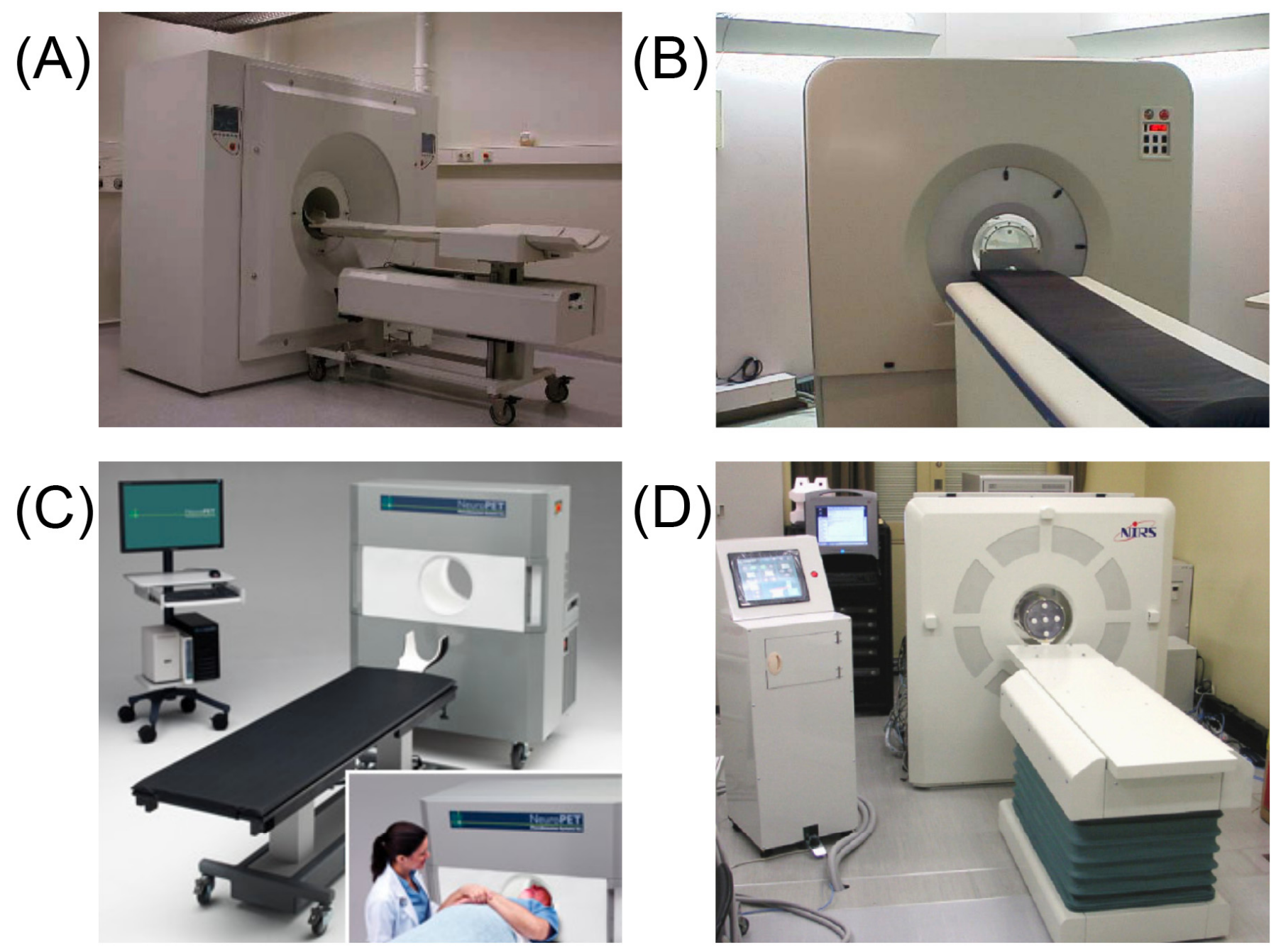

Fig. (21). Brain-dedicated PET scanners. (A) Siemens HRRT. (B) Philips G-PET. (C) PhotoDetection Systems NeuroPET. (D) NIRS jPETD4. (Reprinted from [77, 79, 102]; Courtesy of PhotoDetection Systems). 
PET/CT scanners remains open because molecular brain imaging agents are a topic of active research, particularly for the early accurate diagnosis of neurodegenerative disorders [82-84]. NeuroPET ${ }^{\text {TM }}$ of PhotoDetection Systems Inc. shown in Fig. (21C) is a brain-dedicated PET system that is commercially available.

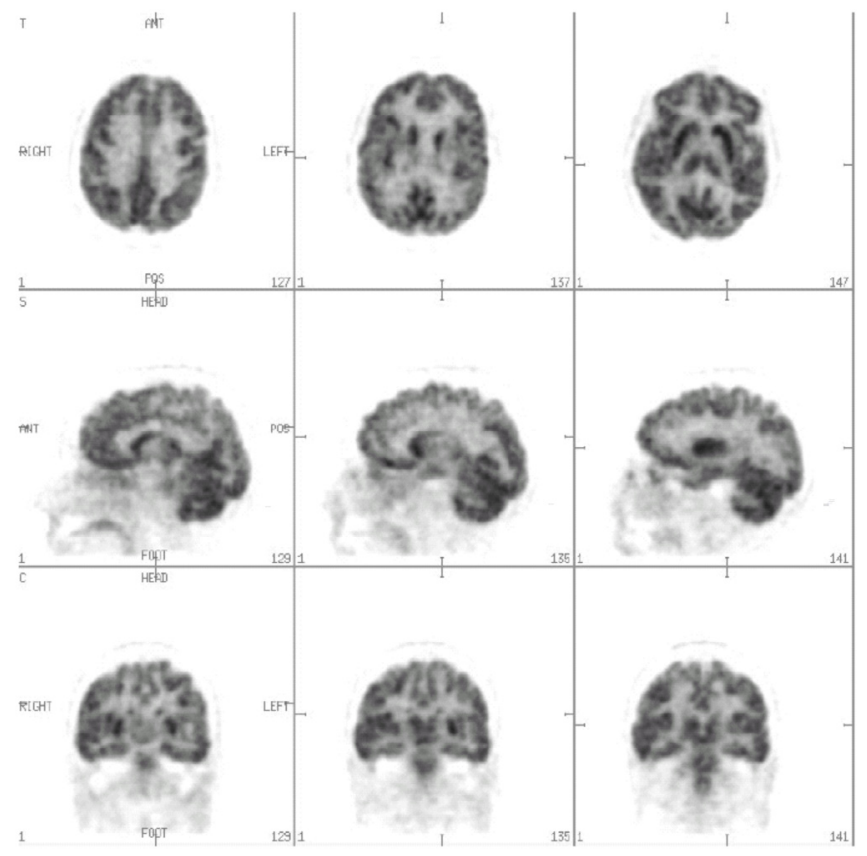

Fig. (22). PET images acquired using brain-dedicated cameras. (Reprinted from [77]).

\section{Breast-Dedicated Positron Imaging Systems}

Breast-dedicated positron imaging systems have received much attention because breast caner is the most common cancer in woman, and its incidence continues to increase. In addition, an optimized design for breast imaging is required. Although early diagnosis is important in breast cancer, whole-body PET/CT examinations are limited for the detection of small breast masses, and the spatial resolutions of breast images acquired by conventional whole-body $\mathrm{PET} / \mathrm{CT}$ are appreciably worse than vendor specifications measured at the center of gantries because breasts are placed at the periphery of PET FOVs. Accordingly, the sensitivity of PET for the detection of breast masses smaller than $1 \mathrm{~cm}$ is less than $60 \%$.

Breast-dedicated scanners commonly involve placing the detectors close to breasts, but several other design ideas have been suggested (Fig. 23) [85]. Positron emission mammography (PEM) systems consist of two parallel detectors that do not rotate, and these provide slice data parallel to detectors using focal plane tomographic techniques (or limited-angle tomography) (Fig. 24) [86, 87]. Usually, the breasts are compressed by the detector head to spread out breast tissues and provide a view similar to that of X-ray mammography [88]. This open geometry allows detector distance to be adjusted for scanning breasts of different sizes and for performing needle biopsies while patients lay or sit in the scanner [21, 89]. DOI measurement would not be essential for PEM cameras because the incidence angles of the gamma rays relative to the long axes of scintillation crystals are relatively small. Naviscan is the first commercially available PEM scanner that provides a 2 $\mathrm{mm}$ spatial resolution in plane. The shortcomings of these PEM systems using focal plane tomography that received most attention are the significant image blurring that occurs along the axis perpendicular to the detector plane and the broad background noise, which reduces image contrast [21, 89]. Although an iterative reconstruction algorithm that provides accurate modeling of statistical noise and detector response was devised to solve the above problems due to limited angular coverage of PEM systems with two parallel detectors, convergence required several hours depending on data size [87].
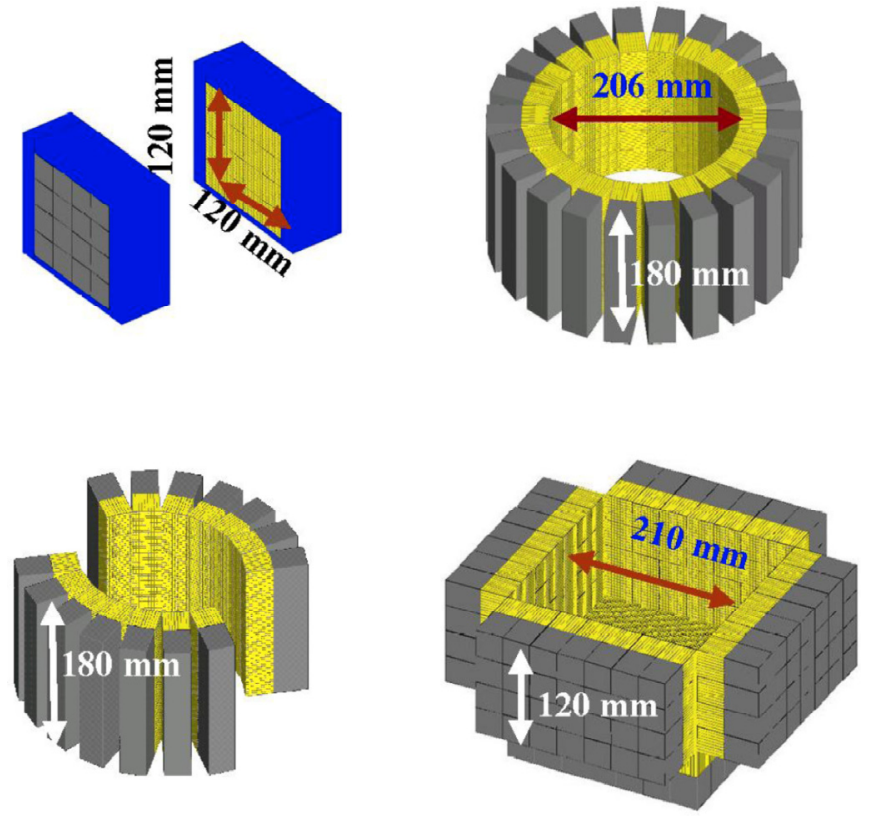

Fig. (23). Various design concepts for breast-dedicated positron imaging devices. (Reprinted from [85]).

Widening the angular coverage by rotating the planar detectors or utilizing a full ring or rectangular camera geometry provide the most direct ways of coping with the limitations of limited-angle tomography and of providing isotropic resolution in all three dimensions. High geometrical detection efficiency can be achieved using the full ring and the rectangular designs, and simulation studies have shown that the count rate performances of these cameras are better than those of cameras with two parallel detectors [85, 90]. On the other hand, rotating planar detectors allow easier access to the breast for biopsy, although some photons loss occurs at gaps between the detectors [89].

Use of stationary partial ring (or split ring) detectors provides a possible compromise between the approaches mentioned above, and the distortions and artifacts associated with the limited angular coverage of the partial ring design could be significantly reduced by incorporating TOF information or Bayesian reconstruction algorithms [21, 91].

\section{Hybrid Breast PET Systems}

Multi-modal combinations of breast-dedicated imaging systems are being actively researched. Preliminary clinical results obtained using a prototype breast-dedicated PET/CT scanner were recently reported $[92,93]$. In this study, a conebeam CT consisting of an X-ray tube and a flat-panel 


\section{(A)}

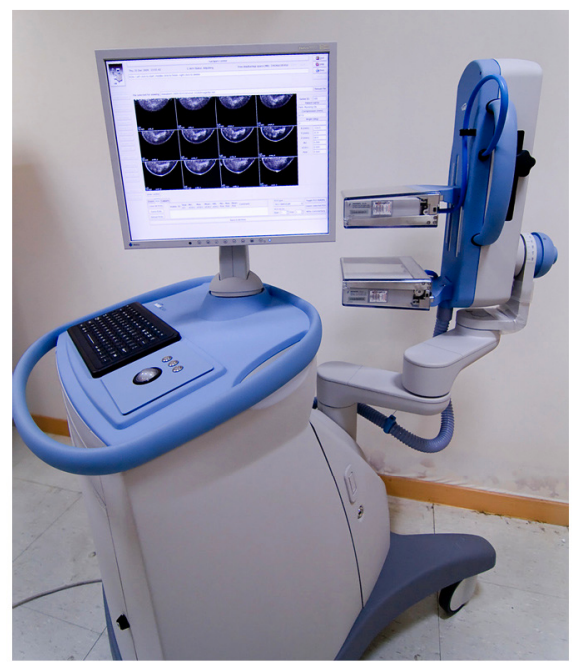

(B)

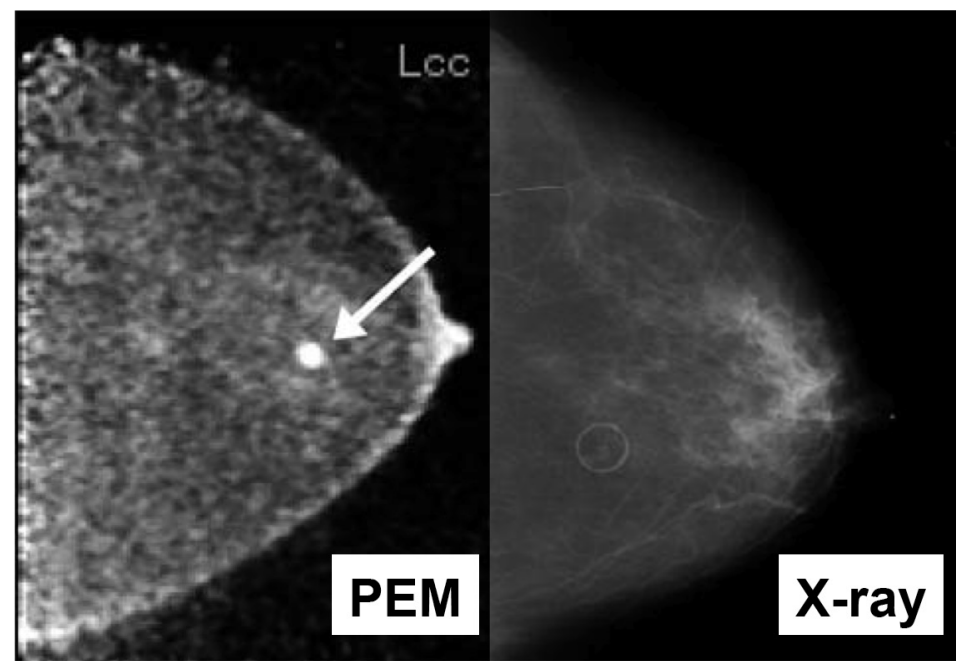

Fig. (24). Positron emission mammography (PEM). (A) Device. (B) Image (Reprinted from [88]).

detector was integrated with a rotating dual-head PET (Fig. 25). Dedicated breast CT using a digital flat-panel detector has potential advantages over conventional X-ray mammography and whole-body CT, namely, it provides clearer images of suspected lesions by overcoming the tissue superposition problem of mammography and higher spatial resolutions and tumor contrast than conventional CT [94,
95]. Diagnostic confidence in and the quantitative accuracy of PET assessments is possibly also enhanced by the anatomical and functional information provided by breastdedicated CT.

The Brookhaven National Laboratory group is currently developing a simultaneous breast PET/MRI. Synergistic effects are anticipated due to the combination of high
(A)

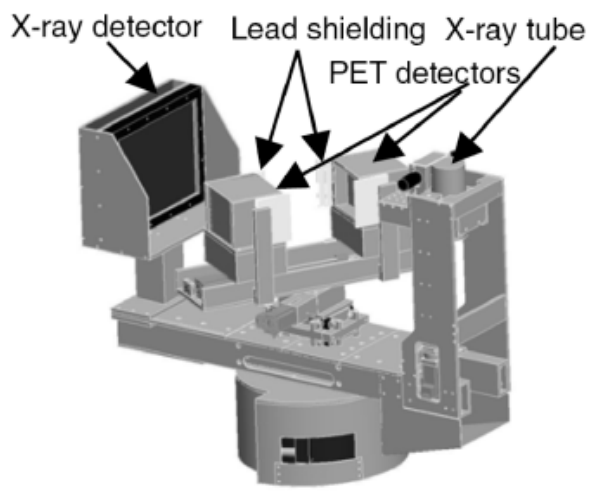


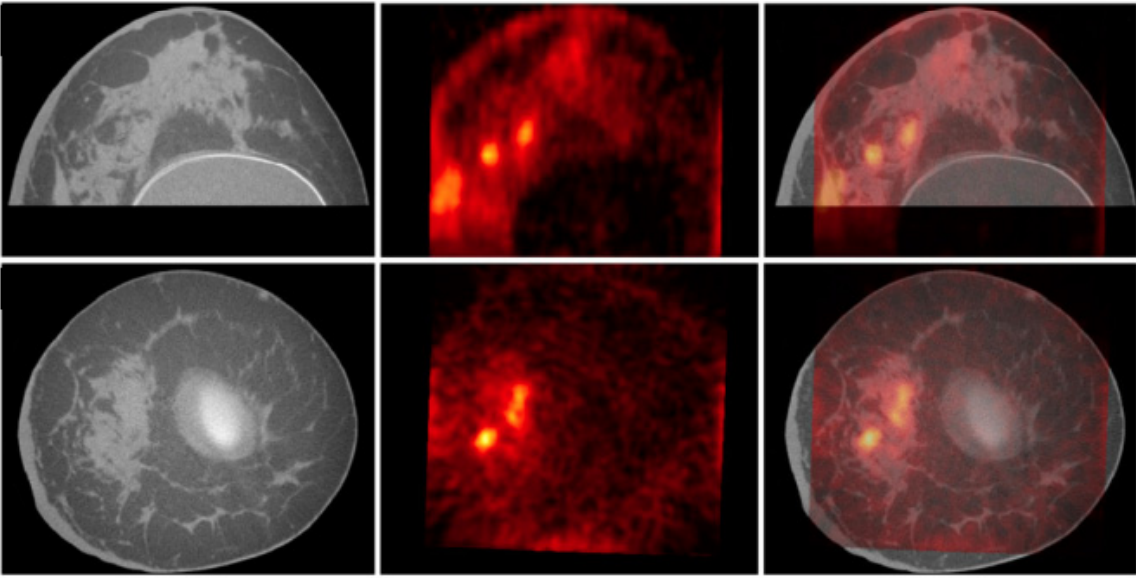

Fig. (25). Hybrid breast imaging system (combination of PEM and x-ray CT). (A) Device. (B) Images. (Reprinted from [92, 93]). 
sensitivity and accurate anatomical information provided by MRI and the high specificity and unique biochemical information provided by PET [96]. For this purpose, they have incorporated their APD-based PET detectors, which were developed for conscious rat brain imaging (RatCAP scanner), with breast specific MRI scanners manufactured by Aurora Imaging Technology (Fig. 26).

(A)

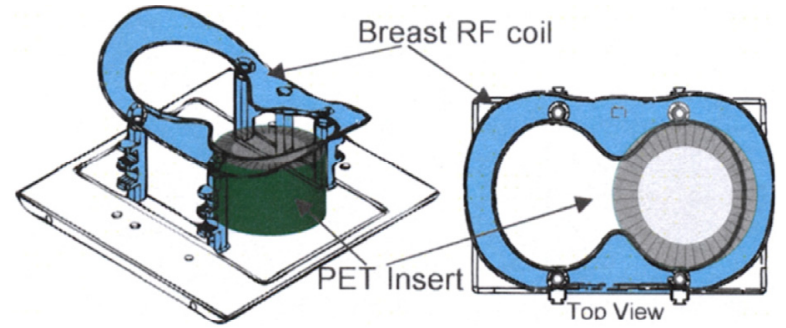

(B)

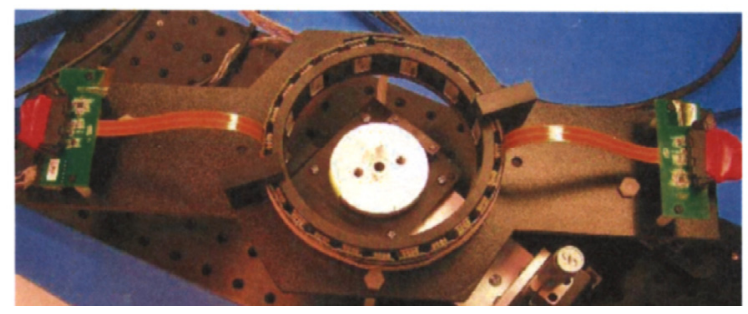

Fig. (26). Breast PET/MRI (A) Conceptual drawing of PET insert and MR coil. (B) Prototype PET insert. (Reprinted from [96]).

In addition to the technical advances achieved, new radiopharmaceuticals that can overcome the limited diagnostic performance of $\left[{ }^{18} \mathrm{~F}\right] \mathrm{FDG}$ will undoubtedly play important roles in the wider use of breast-specific PET scanners. Actually, PET radiotracers promising for breast cancer imaging are being actively investigated. For example, $\left[{ }^{18} \mathrm{~F}\right]$ fluoroestradiol (FES; an estrogen receptor antagonist) and radioligands for HER2 receptor (human epidermal growth factor receptor type 2) are candidate radiopharmaceuticals for breast cancer imaging $[97,98]$.

\section{(A)}

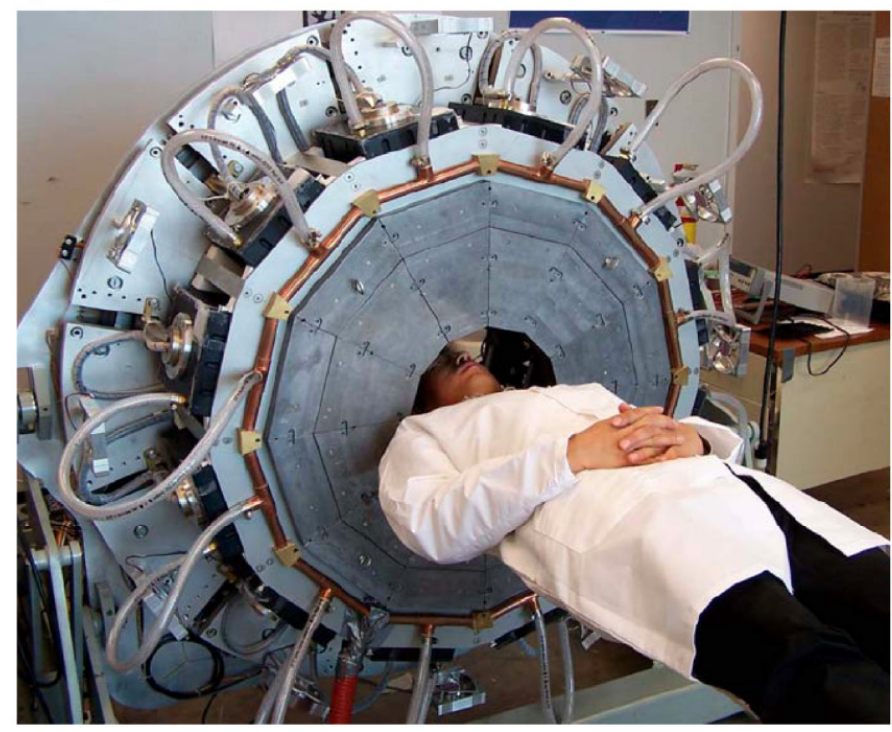

\section{Transformable PET}

In parallel with approaches targeting the use of organspecific PET scanners, efforts are also being made to develop a 'transformable' PET system. In particular, a group at the University of Texas led by W-H. Wong have developed a transformable PET camera $[99,100]$ that can adaptively change PET ring geometry to suit the dimensions of the object imaged (Fig. 27). This approach involves 12 rectangular BGO detector modules with a 13 x $21 \mathrm{~cm}^{2}$ detection area that can be rotated by $90^{\circ}$ about their individual axis and translated in a radial direction to change the ring diameter. Therefore, this camera can have two ring geometries, namely, a diameter of $83 \mathrm{~cm}$ and an axial FOV of $13 \mathrm{~cm}$ for whole-body imaging or a diameter of $54 \mathrm{~cm}$ and axial FOV of $21 \mathrm{~cm}$ for brain/breast imaging. Although the relatively short axial FOV in whole-body mode and the longterm stability of fine mechanical movements are matters of clinical and technical concern, the concept of a transformable PET geometry is attractive, because it allows optimized PET images to be obtained for specific applications.

\section{Large-Bore PET}

There is also a need to increase the ring diameters of PET and CT gantries. Radiation therapy planning procedures require an imaging system with a sufficiently large bore to provide flexibility with respect to patient position. The Philips GEMINI TF Big bore PET/CT has a bore size of 85 $\mathrm{cm}$ which is larger than the standard TOF PET/CT scanner (70 $\mathrm{cm}$ bore size) offered by Philips (Fig. 28). To increase the bore size, the inner diameter of annular lead shielding at the axial end of the PET ring is changed from $71.2 \mathrm{~cm}$ to $86.4 \mathrm{~cm}$.

\section{DISCUSSION}

In this paper, recent advances in PET and related hybrid imaging systems used for clinical and research purposes has been reviewed. The improved technologies in PET hardware

\section{(B)}

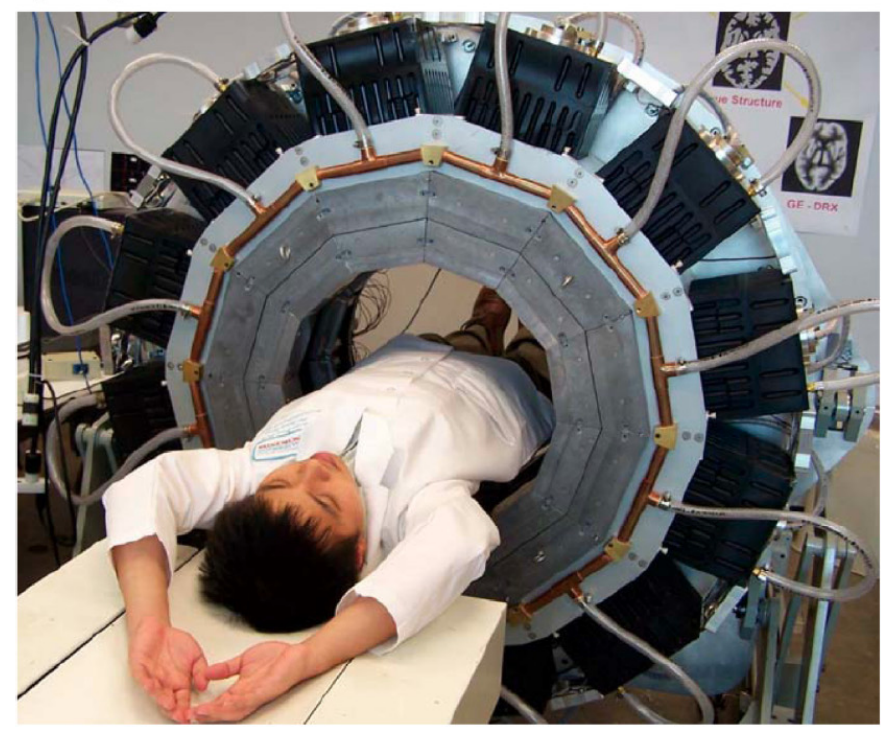

Fig. (27). Transformable PET (A) Brain mode. (B) Whole-body mode. (Reprinted from [100]). 
and software have been leaded to the significant enhancements in the physical performances of the PET devices. However, it seems that there is no enough convincing data yet to clearly elucidate how these enhancements has contributed to the increased reliability of the clinical interpretation and research outcomes. Further more investigations are expected to justify the biological benefits of these technical advances although, sometimes, they are not easily measurable.

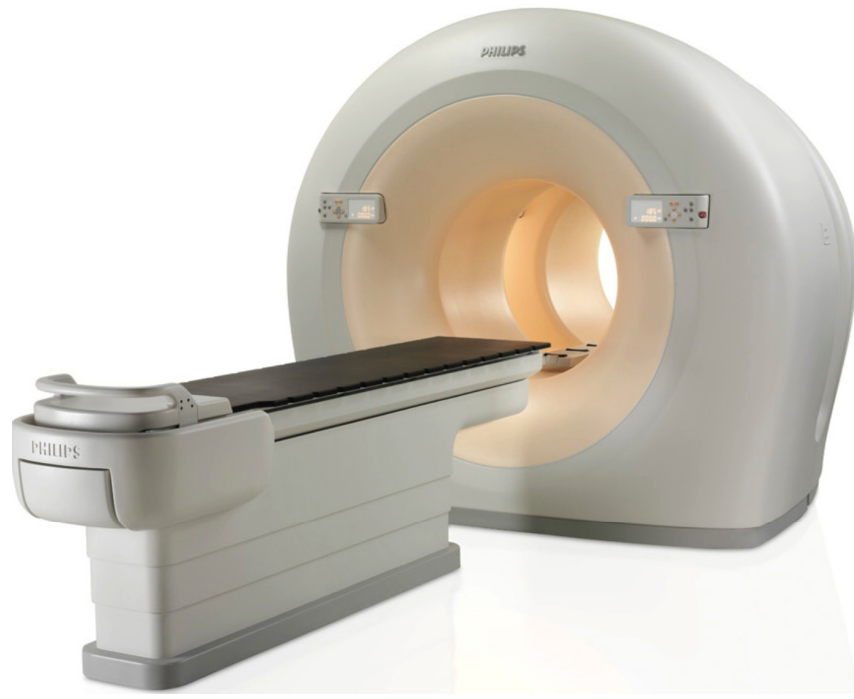

Fig. (28). Large Bore PET (Courtesy of Philips Medical).

It is highly likely that PET/MRI will play its important roles in the biomedical practices and investigations as a compensatory or alternative hybrid PET imaging modality to the conventional PET/CT. However the technologies illustrated in this paper have been mostly developed and validated using the PMT-based PET detectors under no influence of strong external magnetic field. Because the semiconductor photo-sensors or optical fibers employed in the PET/MRI devices usually involve the alternation of detector characteristics, such as the energy, timing and spatial resolutions and signal to noise ratio of output signals, the feasibility of these technologies should be manifested for PET/MRI devices through the further investigations.

Although the application-specific PET cameras have longstanding history of investigations, the commercialization of these devices is on the early stage. In addition, their success in the market cannot be easily predicted because there are many non-technical factors that influence the fate of biomedical devices. Outstanding physical and clinical performances as well as the minimized additional cost and improved patient's convenience would be the least requirements to make their commercial success.

\section{ACKNOWLEDGEMENTS}

This work was supported by grants from the Atomic Energy R\&D Program (2008-03852, 2009-0078724) and the World Class University Program (R32-10142) through the Korea Science and Engineering Foundation funded by the Korean Ministry of Education, Science, and Technology.

\section{REFERENCES}

[1] de Geus-Oei LF, Vriens D, van Laarhoven HW, van der Graaf WT, Oyen WJ. Monitoring and predicting response to therapy with $18 \mathrm{~F}-$
FDG PET in colorectal cancer: a systematic review. J Nucl Med 2009; 50(Suppl 1): 43S-54S.

[2] Hicks RJ. Role of 18F-FDG PET in assessment of response in nonsmall cell lung cancer. J Nucl Med 2009; 50(Suppl 1): 31S-42S.

[3] Lucignani G. Clinical applications of PET amyloid imaging: an update. Eur J Nucl Med Mol Imaging 2009; 36(7): 1185-90.

[4] Pomper MG, Lee JS. Small animal imaging in drug development. Curr Pharm Des 2005; 11(25): 3247-72.

[5] Beyer T, Townsend DW, Brun T, et al. A combined PET/CT scanner for clinical oncology. J Nucl Med 2000; 41(8): 1369-79.

[6] Townsend DW. Dual-modality imaging: combining anatomy and function. J Nucl Med 2008; 49(6): 938-55.

[7] Schlemmer HP, Pichler BJ, Schmand M, et al. Simultaneous MR/PET imaging of the human brain: feasibility study. Radiology 2008; 248(3): 1028-35.

[8] Delso G, Ziegler S. PET/MRI system design. Eur J Nucl Med Mol Imaging 2009; 36(Suppl 1): S86-92.

[9] Wehrl HF, Judenhofer MS, Wiehr S, Pichler BJ. Pre-clinical PET/MR: technological advances and new perspectives in biomedical research. Eur J Nucl Med Mol Imaging 2009; 36(Suppl 1): S56-68.

[10] Lee JS, Hong SJ, Geiger-mode avalanche photodiodes for PET/MRI. In: Iniewski K, Eds. Electronic Circuits for Radiation Detection. CRC Press LLC; 2010:179-200.

[11] Melcher CL, Spurrier MA, Eriksson L, et al. Advances in the scintillation performance of LSO: Ce single crystals. IEEE Trans Nucl Sci 2003; 50(4): 762-6.

[12] Usui T, Shimizu S, Shimura $\mathrm{N}$, et al. $60 \mathrm{~mm}$ diameter Lu0.4Gd1.6SiO5: Ce (LGSO) single crystals and their improved scintillation properties. IEEE Trans Nucl Sci 2007; 54(1): 19-22.

[13] Kadrmas DJ, Casey ME, Conti M, Jakoby BW, Lois C, Townsend DW. Impact of time-of-flight on PET tumor detection. J Nucl Med 2009; 50(8): 1315-23.

[14] Conti M. State of the art and challenges of time-of-flight PET. Phys Med 2009; 25(1):1-11.

[15] Moses WW. Time of flight in PET revisited. IEEE Trans Nucl Sci 2003; 50(5): 1325-30.

[16] Karp JS, Surti S, Daube-Witherspoon ME, Muehllehner G. Benefit of time-of-flight in PET: experimental and clinical results. J Nucl Med 2008; 49(3):462-70.

[17] Surti S, Karp JS. Experimental evaluation of a simple lesion detection task with time-of-flight PET. Phys Med Biol 2009; 54(2): 373-84.

[18] Vunckx K, Zhou L, Matej S, Defrise M, Nuyts J. Fisher information-based evaluation of image quality for time-of-flight PET. IEEE Trans Med Imaging 2009; 29(2): 311-21.

[19] Pentlow KS, Graham MC, Lambrecht RM, Cheung NKV, Larson SM. Quantitative imaging of I-124 using positron emission tomography with applications to radioimmunodiagnosis and radioimmunotherapy. Med Phys 1991; 18(3): 357-66.

[20] Surti S, Scheuermann R, Karp JS. Correction technique for cascade gammas in I-124 imaging on a fully-3D, time-of-flight PET scanner. IEEE Trans Nucl Sci 2009; 56(3): 653-60.

[21] Surti S, Karp JS. Design considerations for a limited angle, dedicated breast, TOF PET scanner. Phys Med Biol 2008; 53(11): 2911-21.

[22] Crespo P, Shakirin G, Fiedler F, Enghardt W, Wagner A. Direct time-of-flight for quantitative, real-time in-beam PET: a concept and feasibility study. Phys Med Biol 2007; 52(23): 6795-811.

[23] Vandenberghe S, Lemahieu I. System characteristics of simulated limited angle TOF PET. Nucl Instrum Meth A 2007; 571(1-2): 480-3.

[24] Shao Y, Cherry SR, Farahani K, et al. Simultaneous PET and MR imaging. Phys Med Biol 1997; 42(10): 1965-70.

[25] Marsden PK, Strul D, Keevil SF, Williams SC, Cash D. Simultaneous PET and NMR. Br J Radiol 2002; 75: S53-9.

[26] Raylman RR, Majewski S, Lemieux SK, et al. Simultaneous MRI and PET imaging of a rat brain. Phys Med Biol 2006; 51(24): 6371-9.

[27] Lucas AJ, Hawkes RC, Ansorge RE, et al. Development of a combined microPET-MR system. Technol Cancer Res Treat 2006; 5(4): $337-41$.

[28] Lucignani G. Time-of-flight PET and PET/MRI: recurrent dreams or actual realities? Eur J Nucl Med Mol Imaging 2006; 33(8): 96971. 
[29] Pichler BJ, Judenhofer MS, Catana C, et al. Performance test of an LSO-APD detector in a 7-T MRI scanner for simultaneous PET/MRI. J Nucl Med 2006; 47(4): 639-47.

[30] Woody C, Schlyer D, Vaska P, et al. Preliminary studies of a simultaneous PET/MRI scanner based on the RatCAP small animal tomograph. Nucl Instrum Meth A 2007; 571(1-2): 102-5.

[31] Del Guerra A, Belcari N, Bisogni MG, Llosa G, Marcatili S, Moehrs S. Advances in position-sensitive photodetectors for PET applications. Nucl Instrum Meth A 2009; 604(1-2): 319-22.

[32] Lee JS, Ito $\mathrm{M}$, $\operatorname{Sim} \mathrm{KS}$, et al. Investigation of solid-state photomultipliers for positron emission tomography scanners. J Korean Phys Soc 2007; 50(5): 1332-9.

[33] Hong SJ, Song IC, Ito M, et al. An investigation into the use of Geiger-mode solid-state photomultipliers for simultaneous PET and MRI acquisition. IEEE Trans Nucl Sci 2008; 55(3): 882-8.

[34] Kim CL, Wang GC, Dolinsky S. Multi-pixel photon counters for TOF PET detector and its challenges. 2008 IEEE Nuclear Science Symposium Conference Record 2008: 3586-90.

[35] Panin VY, Kehren F, Michel C, Casey M. Fully 3-D PET reconstruction with system matrix derived from point source measurements. IEEE Trans Med Imaging 2006; 25(7): 907-21.

[36] Siddon RL. Fast calculation of the exact radiological path for a three-dimensional CT array. Med Phys 1985; 12(2): 252-5.

[37] Johnson CA, Yan YC, Carson RE, Martino RL, Daubewitherspoon ME. A system for the $3 \mathrm{D}$ reconstruction of retracted-septa PET data using the EM algorithm. IEEE Trans Nucl Sci 1995; 42(4): 1223-7.

[38] Schretter C. A fast tube of response ray-tracer. Med Phys 2006; 33(12): 4744-8.

[39] Qi JY, Leahy RM, Cherry SR, Chatziioannou A, Farquhar TH. High-resolution 3D Bayesian image reconstruction using the microPET small-animal scanner. Phys Med Biol 1998; 43(4): 1001-13.

[40] Rafecas M, Mosler B, Dietz M, et al. Use of a Monte Carlo-based probability matrix for 3-D iterative reconstruction of MADPET-II data. IEEE Trans Nucl Sci 2004; 51(5): 2597-605.

[41] Yamaya $\mathrm{T}$, Hagiwara N, Obi $\mathrm{T}$, et al. Preliminary resolution performance of the prototype system for a 4-layer DOI-PET scanner: jPET-D4. IEEE Trans Nucl Sci 2006; 53(3): 1123-8.

[42] Reader AJ, Julyan PJ, Williams H, Hastings DL, Zweit J. EM algorithm system modeling by image-space techniques for PET reconstruction. IEEE Trans Nucl Sci 2003; 50(5): 1392-7.

[43] Kim JS, Lee JS, Im KC, et al. Performance measurement of the microPET focus 120 scanner. J Nucl Med 2007; 48(9): 1527-35.

[44] Yao R, Seidel J, Johnson CA, Daube-Witherspoon ME, Green MV, Carson RE. Performance characteristics of the 3-D OSEM algorithm in the reconstruction of small animal PET images. Ordered-subsets expectation-maximixation. IEEE Trans Med Imaging 2000; 19(8): 798-804.

[45] Bing B, Smith AM. Fast 3D iterative reconstruction of PET images using PC graphics hardware. 2006 IEEE Nuclear Science Symposium Conference Record 2006; 5: 2787-2790.

[46] Schellmann M, Gorlatch S, Meiländer D, et al. Parallel medical image reconstruction: from graphics processors to grids. Lect Notes Comput Sc 2009; 5698: 457-73.

[47] Pratx G, Chinn G, Olcott PD, Levin CS. Fast, accurate and shiftvarying line projections for iterative reconstruction using the GPU. IEEE Trans Med Imaging 2009; 28(3): 435-45.

[48] Ha WS, Kim SM, Park MJ, Lee DS, Lee JS. A study on GPUbased iterative ML-EM reconstruction algorithm for emission computed tomographic imaging systems. Nucl Med Mol Imaging 2009; 43(5): 459-67.

[49] Park MJ, Lee JS, Kim SM, Kang JY, Lee DS, Park KS. Preliminary study on the enhancement of reconstruction speed for emission computed tomography using parallel processing. Nucl Med Mol Imaging 2009;43(5): 443-50.

[50] Yamaya T, Hagiwara N, Obi T, et al. Transaxial system models for jPET-D4 image reconstruction. Phys Med Biol 2005; 50(22): 533955.

[51] McElroy DP, Hoose M, Pimpl W, Spanoudaki V, Schuler T, Ziegler SI. A true singles list-mode data acquisition system for a small animal PET scanner with independent crystal readout. Phys Med Biol 2005; 50(14): 3323-35.

[52] Schmand M, Eriksson L, Casey ME, et al. Performance results of a new DOI detector block for a High Resolution PET-LSO Research Tomograph HRRT. IEEE Trans Nucl Sci 1998; 45(6): 3000-6.
[53] Seidel J, Vaquero JJ, Siegel S, Gandler WR, Green MV. Depth identification accuracy of a three layer phoswich PET detector module. IEEE Trans Nucl Sci 1999; 46(3): 485-90.

[54] Wang Y, Seidel J, Tsui BM, Vaquero JJ, Pomper MG. Performance evaluation of the GE healthcare eXplore VISTA dual-ring smallanimal PET scanner. J Nucl Med 2006; 47(11): 1891-900.

[55] Fontaine R, Belanger F, Viscogliosi N, et al. The hardware and signal processing architecture of LabPET (TM), a small animal apd-based digital pet scanner. IEEE Trans Nucl Sci 2009; 56(1): 3 9 .

[56] Streun M, Brandenburg G, Larue H, et al. Pulse shape discrimination of LSO and LuYAP scintillators for depth of interaction detection in PET. IEEE Trans Nucl Sci 2003; 50(3): 344-7.

[57] Hong SJ, Il Kwon S, Ito M, et al. Concept verification of threelayer DOI detectors for small animal PET. IEEE Trans Nucl Sci 2008; 55(3): 912-7.

[58] Liu H, Omura T, Watanabe M, Yamashita T. Development of a depth of interaction detector for gamma-rays. Nucl Instrum Meth A 2001; 459(1-2): 182-90.

[59] Zhang N, Thompson CJ, Cayouette F, Jolly D, Kecani S. A prototype modular detector design for high resolution positron emission mammography imaging. IEEE Trans Nucl Sci 2003; 50(5): 1624-9.

[60] Mizuta T, Kitamura K, Iwata H, et al. Performance evaluation of a high-sensitivity large-aperture small-animal PET scanner: ClairvivoPET. Ann Nucl Med 2008; 22(5): 447-55.

[61] Ito M, Hong B, Hong SJ, et al. A novel four-layer configuration for animal PET scanners with depth of interaction (DOI) measurement. J Nucl Med 2007; 48(Suppl 2): 40P.

[62] Murayama H, Ishibashi H, Uchida H, Omura T, Yamashita T. Depth encoding multicrystal detectors for PET. IEEE Trans Nucl Sci 1998; 45(3):1152-7.

[63] Inadama $\mathrm{N}$, Murayama $\mathrm{H}$, Hamamoto $\mathrm{M}$, et al. 8-layer DOI encoding of 3-dimensional crystal array. 2005 IEEE Nuclear Science Symposium Conference Record 2005; 5: 2766-9.

[64] Moses WW, Derenzo SE, Melcher CL, Manente RA. A roomtemperature LSO/PIN photodiode PET detector module that measures depth of interaction. IEEE Trans Nucl Sci 1995; 42(4): 1085-9.

[65] Shao Y, Silverman RW, Farrell R, et al. Design studies of a high resolution PET detector using APD arrays. IEEE Trans Nucl Sci 2000; 47(3): 1051-7.

[66] Yang YF, Wu YB, Qi JY, et al. A prototype PET scanner with DOI-encoding detectors. J Nucl Med 2008; 49(7): 1132-40.

[67] Miyaoka RS, Lewellen TK, Yu JH, McDaniel DL. Design of a depth of interaction (DOI) PET detector module. IEEE Trans Nucl Sci 1998; 45(3): 1069-73.

[68] Lewellen TK, Janes M, Miyaoka RS, editors. DMice-a depth-ofinteraction detector design for PET scanners. 2004 IEEE Nuclear Science Symposium Conference Record 2004; 4: 2388-92.

[69] Ito M, Lee JS, Park MJ, Sim KS, Hong SJ. Design and simulation of a novel method for determining depth-of-interaction in a PET scintillation crystal array using a single-ended readout by a multianode PMT. Phys Med Biol. 2010; 55(13): 3827-3841.

[70] Tomitani T, Futami Y, Iseki Y, et al. Depth encoding of point-ofinteraction in thick scintillation cameras. 1999 IEEE Nuclear Science Symposium Conference Record 1999; 3: 1182-6.

[71] Joung J, Miyaoka RS, Lewellen TK. cMiCE: a high resolution animal PET using continuous LSO with a statistics based positioning scheme. Nucl Instrum Meth A. 2002; 489(1-3): 584-98.

[72] Ling T, Lewellen TK, Miyaoka RS. Improving the intrinsic spatial resolution performance of the continuous miniature crystal element (cMiCE) detector. 2007 IEEE Nuclear Science Symposium Conference Record 2007; 6: 4308-13.

[73] Chung YH, Lee SJ, Baek $\mathrm{CH}$, Choi Y. New design of a quasimonolithic detector module with DOI capability for small animal pet. Nucl Instrum Meth A 2008; 593(3): 588-91.

[74] Lee SJ, Baek CH, Chung YH, Choi Y, Lee W. A cross-stack quasimonolithic detector with DOI capability for a small animal PET. J Korean Phys Soc 2009; 54(6): 2429-34.

[75] Watanabe M, Shimizu K, Omura T, et al. A new high-resolution PET scanner dedicated to brain research. IEEE Trans Nucl Sci 2002; 49(3): 634-9. 
[76] Braem A, Llatas MC, Chesi E, et al. Feasibility of a novel design of high resolution parallax-free Compton enhanced PET scanner dedicated to brain research. Phys Med Biol 2004; 49(12): 2547-62.

[77] Surti S, Karp JS, Adam LE, Muehllehner G. Performance measurements for the GSO-based brain PET camera (G-PET). 2001 IEEE Nuclear Science Symposium Conference Record 2001; 2: $1109-14$.

[78] Wienhard K, Schmand M, Casey ME, et al. The ECAT HRRT: Performance and first clinical application of the new high resolution research tomograph. IEEE Trans Nucl Sci 2002; 49(1): $104-10$.

[79] de Jong HW, van Velden FH, Kloet RW, Buijs FL, Boellaard R, Lammertsma AA. Performance evaluation of the ECAT HRRT: an LSO-LYSO double layer high resolution, high sensitivity scanner. Phys Med Biol 2007; 52(5):1505-26.

[80] Heiss WD, Habedank B, Klein JC, et al. Metabolic rates in small brain nuclei determined by high-resolution PET. J Nucl Med 2004; 45(11): 1811-5.

[81] van Velden FH, Kloet RW, van Berckel BN, et al. HRRT versus HR+ human brain PET studies: an interscanner test-retest study. J Nucl Med 2009; 50(5): 693-702.

[82] Klunk WE, Engler H, Nordberg A, et al. Imaging brain amyloid in Alzheimer's disease with Pittsburgh Compound-B. Ann Neurol 2004; 55(3): 306-19.

[83] Small GW, Kepe V, Ercoli LM, et al. PET of brain amyloid and tau in mild cognitive impairment. N Engl J Med 2006; 355(25): 265263.

[84] Lucignani G. Clinical applications of PET amyloid imaging: an update. Eur J Nucl Med Mol Imaging 2009; 36(7): 1185-90.

[85] Bowen SL, Yang Y, Wu Y, et al. Monte Carlo simulation study of several camera designs for the PET component of a dedicated breast PET/CT scanner. 2006 IEEE Nuclear Science Symposium Conference Record 2006; 6: 3920-4.

[86] Muehllehner G. Letter: Resolution limit of positron cameras. J Nucl Med 1976; 17(8): 757-8.

[87] Moses WW, Qi J. Fundamental limits of positron emission mammography. Nucl Instrum Meth A 2003; 497(1): 82-9.

[88] Berman CG. Recent advances in breast-specific imaging. Cancer Control 2007; 14(4): 338-49.

[89] Raylman RR, Majewski S, Smith MF, et al. The positron emission mammography/tomography breast imaging and biopsy system (PEM/PET): design, construction and phantom-based measurements. Phys Med Biol 2008; 53(3): 637-53.
[90] Qi JY, Kuo C, Huesman RH, Klein GJ, Moses WW, Reutter BW. Comparison of rectangular and dual-planar positron emission mammography scanners. IEEE Trans Nucl Sci 2002; 49(5): 208996.

[91] Kitamura K, Kudo H. MAP-EM reconstruction using uniform background template for limited-angle PEM. 2008 IEEE Nuclear Science Symposium Conference Record. 2008:4172-4.

[92] Bowen SL, Wu Y, Chaudhari AJ, et al. Initial characterization of a dedicated breast PET/CT scanner during human imaging. J Nucl Med 2009; 50(9): 1401-8

[93] Wu Y, Bowen SL, Yang K, et al. PET characteristics of a dedicated breast PET/CT scanner prototype. Phys Med Biol 2009; 54(13): 4273-87.

[94] Karellas A, Vedantham S. Breast cancer imaging: A perspective for the next decade. Med Phys 2008; 35(11):4878-97.

[95] Glick SJ. Breast CT. Annu Rev Biomed Eng 2007; 9: 501-26.

[96] Ravindranath B, Maramraju SH, Junnarkar SS, et al. A simultaneous PET/MRI breast scanner based on the RatCAP. 2008 IEEE Nuclear Science Symposium Conference Record 2008: 46505.

[97] Linden HM, Stekhova SA, Link JM, et al. Quantitative fluoroestradiol positron emission tomography imaging predicts response to endocrine treatment in breast cancer. J Clin Oncol 2006; 24(18):2793-9.

[98] Cheng Z, De Jesus OP, Namavari M, et al. Small-animal PET imaging of human epidermal growth factor receptor type 2 expression with site-specific $18 \mathrm{~F}$-labeled protein scaffold molecules. J Nucl Med 2008; 49(5): 804-13.

[99] Wong WH, Uribe J, Li HD, et al. The design of a high-resolution transformable wholebody PET camera. IEEE Trans Nucl Sci 2002; 49(5): 2079-84.

[100] Zhang Y, Wong W-H, Li Hongdi, et al. Performance characteristics of a high resolution oncologic transformable PET in brain/breast and whole-body modes. 2007 IEEE Nuclear Science Symposium Conference Record 2007; 5: 3684-7.

[101] Lee JS. Nuclear medicine physics. In: Chung J-K, Lee MC, eds. Nuclear Medicine. Korea Medical Book Publisher. 2008.

[102] Kitamura K, Ishikawa A, Mizuta T, Yamaya T, Yoshida E, Murayama $\mathrm{H}$. Detector normalization and scatter correction for the jPET-D4: A 4-layer depth-of-interaction PET scanner. Nucl Instrum Meth A 2007; 571(1-2): 231-4.

(C) Jae Sung Lee; Licensee Bentham Open.

This is an open access article licensed under the terms of the Creative Commons Attribution Non-Commercial License (http://creativecommons.org/licenses/by-nc/ 3.0/) which permits unrestricted, non-commercial use, distribution and reproduction in any medium, provided the work is properly cited. 